\title{
Synthesis and in vitro antimicrobial evaluation of $4 H$-pyrazolopyran, -benzopyran and naphthopyran derivatives of 1H-pyrazole
}

\author{
Nilesh J. Thumar and Manish P. Patel* \\ Department of Chemistry, Sardar Patel University, Vallabh Vidyanagar, 388120, Gujarat, India \\ E-mail: patelmanish1069@yahoo.com
}

\begin{abstract}
A new series of eight derivatives each of 4-pyrazolyl-4H-pyrazolopyran, -benzopyran and sixteen derivatives of naphthopyran has been synthesized by one-pot base-catalyzed cyclocondensation reactions of 1-phenyl-3-(het)aryl-pyrazole-4-carbaldehyde, malononitrile and substituted pyrazolin-5-ones or dimedone or naphthols respectively. All the synthesized compounds were subjected to in vitro antimicrobial screening against a panel of pathogenic strains of bacteria and fungi. Some of the compounds were found to be equipotent or more potent than commercial antibiotics against most of employed strains.
\end{abstract}

Keywords: 4H-Pyran, multi-component reaction, pyrazole-4-carbaldehyde, antimicrobial activity, MIC

\section{Introduction}

The 4H-Pyran nucleus is a fertile source of biologically important molecules possessing a wide spectrum of biological and pharmacological activities, such as antimicrobial, ${ }^{1}$ antiviral, ${ }^{2}$ mutagenicity, ${ }^{3}$ antiproliferative, ${ }^{4}$ sex pheromone, ${ }^{5}$ antitumor, ${ }^{6}$ cancer therapy ${ }^{7}$ and central nervous system activity. ${ }^{8}$ Some of these compounds are widely employed as cosmetics and pigments and as potential biodegradable agrochemicals. ${ }^{9}$ Therefore, the synthesis of such compounds has attracted strong interest.

In recent years, 4-functionally substituted 1,3-diarylpyrazole derivatives have received considerable attention due to their wide range of useful biological properties, which include antimicrobial, ${ }^{10-12}$ anti-inflammatory (COX-2 inhibitor and ulcerogenic activity), ${ }^{11}$ antitubercular, ${ }^{12}$ antitumor, ${ }^{13,14}$ antiangiogenesis, ${ }^{14}$ anti-parasitic ${ }^{15}$ and antiviral activity. ${ }^{16} \mathrm{~A}$ literature survey ${ }^{17}$ revealed that a number of $4 H$-pyran derivatives have been synthesized using various aldehydes but not a single reference has been found where 1,3-diaryl pyrazole-4carbaldehydes are used. Thus, in a view to obtain more biologically potent heterocyclic systems, 
containing therapeutically active moieties pyran and pyrazole, and in continuation of our work ${ }^{18}$ on biologically active heterocyclic compounds, we report herein the synthesis of some new substituted $4 H$-pyrazolopyran, -benzopyran and naphthopyran derivatives of pyrazole via a Multi-Component Reaction (MCR) approach. The constitutions of all the products were confirmed using elemental analysis, FT-IR, ${ }^{1} \mathrm{H}$ NMR and ${ }^{13} \mathrm{C}$ NMR spectroscopy. All synthesized compounds were screened for in vitro antimicrobial activity against eight human pathogens, of which three gram positive bacterial pathogens Streptococcus pneumoniae, Clostridium tetani, Bacillus subtilis, three gram negative bacterial pathogens Salmonella typhi, Vibrio cholerae, Escherichia coli and two fungal pathogens Aspergillus fumigatus and Candida albicans, using broth microdilution MIC (Minimum Inhibitory Concentration) method. ${ }^{19}$

\section{Results and Discussion}

A series of 4-pyrazolyl-4H-pyrazolopyran 6a-h, -benzopyran 7a-h and naphthopyran 8a- h, 9a-h derivatives has been synthesized by one-pot three-component cyclocondensation reaction of 1phenyl-3-(het)aryl-pyrazole-4-carbaldehyde 1a-h, malononitrile $\mathbf{2}$ and substituted pyrazolin-5ones $\mathbf{3}$ or dimedone $\mathbf{4}$ or naphthols $\mathbf{5 a - b}$ respectively, in the presence of piperidine as catalyst. The mixture refluxing under ethanol or acetonitrile gives moderate to good yield (50-76\%) (Scheme 1). A mechanism for the formation of the pyran derivatives is outlined in Scheme 2. The reaction occurs via an in situ initial formation of the heterylidenenitrile, containing the electron-poor $\mathrm{C}=\mathrm{C}$ double bond, from the Knoevenagel condensation between pyrazole-4carbaldehyde and malononitrile by loss of water molecules. Finally, Michael addition of $\mathbf{3}$ or $\mathbf{4}$ or 5 to the initially formed unsaturated nitrile, i.e. nucleophilic attack of hydroxyl moiety to the cyano moiety affords cyclized pyran derivatives 6a-h, 7a-h, 8a-h and 9a-h.

The structures of all the new synthesized compounds were established by ${ }^{1} \mathrm{H} \mathrm{NMR},{ }^{13} \mathrm{C}$ NMR and FT-IR spectral data and molecular weight of some selected compounds confirmed by mass spectrometry. NMR spectroscopy is especially useful to elucidate the structures of products i.e. ${ }^{1} \mathrm{H}$ NMR (DMSO- $d_{6}$ ) spectrum of $\mathbf{6 a}$ exhibited a singlet peak at $\delta 4.59$ for $\mathrm{H} 4$ and $\delta 6.91$ ppm for $\mathrm{NH}_{2}$ of the pyran ring. Aromatic protons of 6a resonate as multiplets at around $\delta 6.98-$ $7.96 \mathrm{ppm}$ and a deshielded aromatic singlet at $\delta 8.31 \mathrm{ppm}$ stands for $\mathrm{H} 5$ of pyrazole ring. A singlet at $\delta 1.79 \mathrm{ppm}$ and $\delta 12.18 \mathrm{ppm}$ stands for methyl and secondary amine of the fused pyrazole ring respectively. ${ }^{13} \mathrm{C}$ NMR of 6 a exhibited a distinctive signal at $\delta 10.4$ ppm for methyl of fused pyrazole ring and $\delta 27.2 \mathrm{ppm}$ for $\mathrm{C} 4$ of the pyran ring. All the aromatic carbons of $6 \mathbf{a}$ showed signals around $\delta 114.5-151.2 \mathrm{ppm}$ in the ${ }^{13} \mathrm{C}$ NMR spectra. Moreover, distinctive signals at $\delta 161.6 \mathrm{ppm}$ for $\mathrm{C} 2, \delta 57.8 \mathrm{ppm}$ for $\mathrm{C} 3, \delta 98.4 \mathrm{ppm}$ for $\mathrm{C} 5, \delta 154.7 \mathrm{ppm}$ for $\mathrm{C} 6$ and $\delta 120.9$ ppm for $\mathrm{CN}$ of pyran ring in the ${ }^{13} \mathrm{C}$ NMR spectra confirms the structure $6 \mathbf{a}$.

The IR spectrum of compound $\mathbf{6 a}$ exhibited characteristic absorption bands at 3410 and $3385 \mathrm{~cm}^{-1}$ (asym. \& sym. str.) for $-\mathrm{NH}_{2}$ and $2210 \mathrm{~cm}^{-1}$ for $-\mathrm{CN}$ functional group respectively. The presence of band around $1220 \mathrm{~cm}^{-1}$ of cyclic ether linkage supports the formation of pyran 
derivative 6a. Further, the structure of selected compounds, $6 \mathbf{e}, 7 \mathbf{7 e}, \mathbf{8 f}$ and $9 \mathrm{~h}$ were confirmed by its mass spectral studies. The mass spectra detected the expected molecular ion signals corresponding to respective molecular formula of synthesized compounds. Mass spectra of compound 6e gave molecular ion peak at $425.1\left(\mathrm{M}^{+}+1\right)$ corresponding to molecular formula $\mathrm{C}_{24} \mathrm{H}_{20} \mathrm{~N}_{6} \mathrm{O}_{2}$.

Similarly, all these compounds were characterized on the basis of spectral studies. All spectroscopic data have been given in experimental section. All the compounds were screened for their antibacterial and antifungal activity.

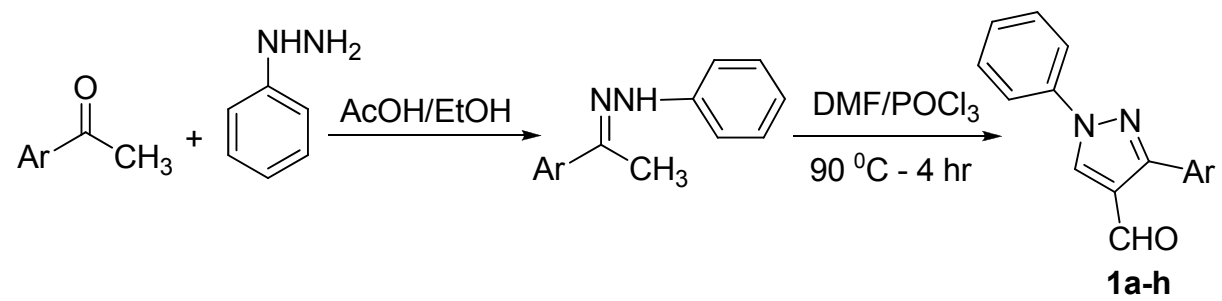<smiles>NC1=C(N)C(c2cn(-c3ccccc3)nc2[Hg])c2c(ccc3ccccc23)O1</smiles>

9a-h<smiles>NC1=C(N)C(c2cn(-c3ccccc3)nc2[Hg]c2cccc3ccccc23)c2ccc3ccccc3c2O1</smiles>

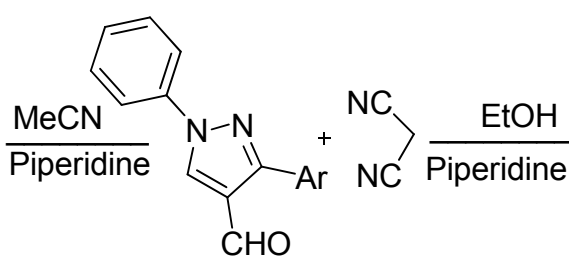

$$
\begin{aligned}
& \text { 1a-h } 2 \\
& \text { Compd } \mathrm{Ar} \\
& \text { a } \quad \mathrm{C}_{6} \mathrm{H}_{5} \\
& \text { b } \quad 4-\mathrm{Br}-\mathrm{C}_{6} \mathrm{H}_{4} \\
& \text { c } \quad 4-\mathrm{Cl}-\mathrm{C}_{6} \mathrm{H}_{4} \\
& \text { d } \quad 4-\mathrm{F}-\mathrm{C}_{6} \mathrm{H}_{4} \\
& \text { e } \quad 4-\mathrm{OCH}_{3}-\mathrm{C}_{6} \mathrm{H}_{4} \\
& \text { f } \quad 4-\mathrm{CH}_{3}-\mathrm{C}_{6} \mathrm{H}_{4} \\
& \begin{array}{cc}
\mathrm{g} & 4-\mathrm{NO}_{2}-\mathrm{C}_{6} \mathrm{H}_{4} \\
\text { h } & \text { 2-thienyl }
\end{array}
\end{aligned}
$$

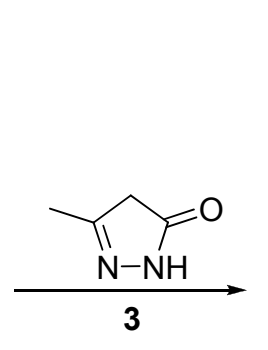<smiles>NNc1ccccc1</smiles><smiles></smiles>

6a-h

Scheme 1. Synthetic pathway for the compounds $6 \mathbf{a}-\mathbf{h}, 7 \mathbf{a}-\mathbf{h}, \mathbf{8 a}-\mathbf{h}$ and $\mathbf{9 a - h}$. 


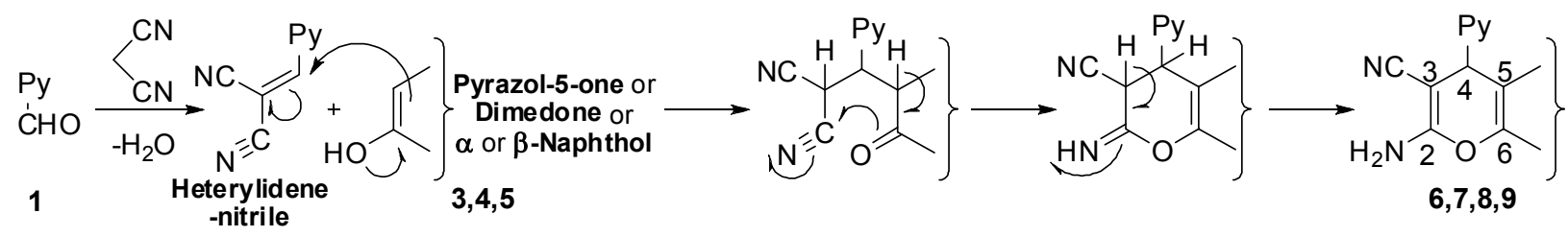

Scheme 2. Plausible mechanistic pathway of the synthesis of pyran derivatives.

\section{Antimicrobial screening}

The in vitro antimicrobial activity of all the synthesized compounds was carried out by broth microdilution method. ${ }^{19}$ Mueller Hinton broth was used as nutrient medium to grow and dilute the compound suspension for the test bacteria and Sabouraud Dextrose broth used for fungal nutrition. Inoculum size for test strain was adjusted to $10^{8} \mathrm{CFUmL}^{-1}$ (Colony Forming Unit per milliliter) by comparing the turbidity. The strains employed for the activity were procured from (MTCC - Micro Type Culture Collection) Institute of Microbial Technology, Chandigarh.

The compounds 6a-h, 7a-h, 8a-h and 9a-h were screened for their antibacterial activity against Bacillus subtilis (MTCC 441), Clostridium tetani (MTCC 449), Streptococcus pneumoniae (MTCC 1936), Escherichia coli (MTCC 443), Salmonella typhi (MTCC 98) and Vibrio cholerae (MTCC 3906) as well as antifungal activity against Aspergillus fumigatus (MTCC 3008) and Candida albicans (MTCC 227). DMSO was used as vehicle to get desired concentration of compounds to test upon microbial strains. The lowest concentration, which showed no visible growth after spot subculture was considered as MIC for each compound. Ampicillin, chloramphenicol, ciprofloxacin, gentamicin and norfloxacin were used as standard antibacterial drugs, whereas griseofulvin and nystatin was used as standard antifungal drugs. The protocols were summarized in Table 1.

The examination of the data (Table 1) reveals that most of the compounds showed excellent antibacterial and antifungal activity when compared with ampicillin and griseofulvin. Against Gram positive pathogen $S$. pneumoniae, compounds $\mathbf{8 b}$ and $9 \mathbf{a}$ were found to be more efficient than chloramphenicol and ciprofloxacin where as, $\mathbf{8 c}$ and $9 \mathrm{~h}$ were more potent, and $\mathbf{7 f}, \mathbf{8 g}$ and $9 \mathrm{f}$ were found to exhibit comparable activity, to ampicillin. The compounds $9 \mathbf{f}$ and $\mathbf{8 g}$ possess comparable activity to ciprofloxacin, and 6a, 6d-g, 7b, 7f, 8a-b, 8d-e, 8h, 9c-d and 9g-h were found equally potent to ampicillin, towards $C$. tetani. The compounds $\mathbf{8 b}$ and $\mathbf{9 a}$ shows better activity where as, $\mathbf{9 h}$ is equally active compared to ciprofloxacin and chloramphenicol and compounds 8c, 8g, 9f shows better activity, and 6a-b, 6d, 7a, 7e-f, 8a, 8d-e, 8h, 9c-d and 9g found equally potent, to ampicillin, against $B$. subtilis. 
Table 1. Antimicrobial activity of the compounds 6a-h, 7a-h, 8a-h and 9a-h

\begin{tabular}{|c|c|c|c|c|c|c|c|c|}
\hline \multirow{3}{*}{ Compds } & \multicolumn{8}{|c|}{ Minimum Inhibitory Concentration (MIC, $\mu g \mathrm{~mL}^{-1}$ ) } \\
\hline & \multicolumn{3}{|c|}{ Gram positive bacteria } & \multicolumn{3}{|c|}{ Gram negative bacteria } & \multicolumn{2}{|c|}{ Fungi } \\
\hline & $\begin{array}{c}\boldsymbol{S} . \boldsymbol{p} . \\
\text { MTCC } 1936\end{array}$ & $\begin{array}{c}\boldsymbol{C} . \boldsymbol{t} . \\
\text { MTCC } 449\end{array}$ & $\begin{array}{c}\text { B. } \boldsymbol{s} . \\
\text { MTCC } 441\end{array}$ & $\begin{array}{c}\boldsymbol{S} . \boldsymbol{t} . \\
\text { MTCC } 98 \\
\end{array}$ & $\begin{array}{c}\boldsymbol{V} . \boldsymbol{c} . \\
\text { MTCC } 3906\end{array}$ & $\begin{array}{c}\boldsymbol{E} . \boldsymbol{c} . \\
\text { MTCC } 443\end{array}$ & $\begin{array}{c}\boldsymbol{A} . \boldsymbol{f} \cdot \\
\text { MTCC } 3008\end{array}$ & $\begin{array}{c}\text { C. } \boldsymbol{a} . \\
\text { MTCC } 227\end{array}$ \\
\hline $\mathbf{6 a}$ & 250 & 250 & 250 & 200 & 500 & 200 & $>1000$ & $\mathbf{5 0 0}$ \\
\hline 6b & 250 & 500 & 250 & 200 & 100 & 125 & $>1000$ & 1000 \\
\hline 6c & 500 & 500 & 1000 & 250 & 500 & 200 & 500 & 500 \\
\hline $6 d$ & 500 & 250 & 200 & 500 & 250 & 250 & $>1000$ & 250 \\
\hline $6 e$ & 250 & 200 & 500 & 250 & 250 & 200 & $>1000$ & 500 \\
\hline $6 f$ & 1000 & 200 & 1000 & 1000 & 250 & 500 & 1000 & 1000 \\
\hline $6 \mathrm{~g}$ & 1000 & 250 & 500 & 1000 & 500 & 500 & $>1000$ & 500 \\
\hline $6 \mathrm{~h}$ & 500 & 500 & 500 & 250 & 500 & 250 & 1000 & 1000 \\
\hline $7 a$ & 1000 & 100 & 250 & 150 & 150 & 250 & $>1000$ & 1000 \\
\hline $7 b$ & 500 & 250 & 1000 & 250 & 500 & 250 & $>1000$ & 500 \\
\hline $7 c$ & 500 & 500 & 500 & 500 & 1000 & 250 & 500 & 500 \\
\hline 7d & 1000 & 1000 & 1000 & 1000 & 500 & 500 & 1000 & 1000 \\
\hline $7 e$ & 500 & 500 & 250 & 250 & 1000 & 250 & 500 & 500 \\
\hline $7 f$ & 100 & 250 & 250 & 150 & 500 & 100 & 1000 & 1000 \\
\hline $7 \mathrm{~g}$ & 500 & 500 & 500 & 500 & 500 & 250 & 500 & 500 \\
\hline $7 \mathrm{~h}$ & 250 & 1000 & 500 & 250 & 1000 & 150 & 500 & 1000 \\
\hline $8 \mathbf{a}$ & 250 & 200 & 250 & 500 & 250 & 500 & 500 & 1000 \\
\hline $8 b$ & 25 & 250 & 25 & 25 & 25 & 62.5 & 1000 & 500 \\
\hline 8c & 62.5 & 500 & 62.5 & 100 & 62.5 & 200 & $>1000$ & 1000 \\
\hline $8 d$ & 250 & 250 & 250 & 150 & 250 & 500 & 500 & 500 \\
\hline $8 e$ & 250 & 250 & 250 & 200 & 250 & 250 & $>1000$ & 1000 \\
\hline $8 f$ & 500 & 500 & 500 & 250 & 500 & 125 & $>1000$ & $>1000$ \\
\hline $8 g$ & 100 & 100 & 100 & 62.5 & 100 & 100 & 1000 & 1000 \\
\hline $8 h$ & 200 & 200 & 200 & 250 & 200 & 100 & 500 & 500 \\
\hline 9a & 25 & 500 & 25 & 50 & 25 & 50 & 1000 & 1000 \\
\hline $9 b$ & 500 & 500 & 500 & 500 & 500 & 500 & 500 & 500 \\
\hline $9 c$ & 200 & 200 & 200 & 62.5 & 200 & 100 & 500 & 1000 \\
\hline 9d & 250 & 250 & 250 & 200 & 250 & 100 & $>1000$ & 500 \\
\hline $9 e$ & 500 & 500 & 500 & 150 & 500 & 125 & $>1000$ & 1000 \\
\hline 9f & 100 & 100 & 100 & 62.5 & 100 & 250 & 500 & 500 \\
\hline $9 \mathrm{~g}$ & 200 & 200 & 200 & 250 & 200 & 500 & 1000 & 500 \\
\hline $9 \mathrm{~h}$ & 50 & 250 & 50 & 25 & 50 & 125 & 500 & 1000 \\
\hline Ampi. & 100 & 250 & 250 & 100 & 100 & 100 & - & - \\
\hline Chlora. & 50 & 50 & 50 & 50 & 50 & 50 & - & - \\
\hline Cipro. & 50 & 100 & 50 & 25 & 25 & 25 & - & - \\
\hline Genta. & 0.5 & 5 & 1 & 5 & 5 & 0.05 & - & - \\
\hline Grise. & - & - & - & - & - & - & 100 & 500 \\
\hline Nyst. & - & - & - & - & - & - & 100 & 100 \\
\hline
\end{tabular}

Ampi.: Ampicillin, Chlora.: Chloramphenicol, Cipro.: Ciprofloxacin, Genta.: Gentamicin, Grise.: Griseofulvin, Nyst.: Nystatin

S. p.: Streptococcus pneumoniae, C. t.: Clostridium tetani, B. s.: Bacillus subtilis, S. t.: Salmonella typhi, V. c.: Vibrio cholerae, E. c.: Escherichia coli, A. f.: Aspergillus fumigatus, $C$. a.: Candida albicans 
Towards Gram negative strain $S$. typhi, compounds $\mathbf{8 b}, 9 \mathbf{h}$ and $9 \mathbf{a}$ were equally active to ciprofloxacin and chloramphenicol where as, compounds $8 \mathrm{~g}, \mathbf{9 c}$ and $9 \mathbf{f}$ were found better active and $8 \mathbf{c}$ is equally active than ampicillin. The Compounds $\mathbf{8 b}, \mathbf{9 a}$ and $\mathbf{9 h}$ found equipotent to ciprofloxacin and chloramphenicol where as, compounds $6 \mathbf{b}, 8 \mathbf{c}, 8 \mathrm{~g}$ and $9 \mathrm{f}$ are comparably active to ampicillin against $V$. cholerae. The compounds $\mathbf{8 b}$ shows better, and $7 \mathbf{f}, \mathbf{8 g}, \mathbf{8 h}, \mathbf{9 c}$ and $9 \mathbf{d}$ were found to exhibit comparable activity to ampicillin towards $E$. coli. The compounds $\mathbf{8 b}$ and 8g were highly active against all the the tested Gram positive and negative pathogens. The remaining compounds showed moderate to good activity to inhibit the growth of bacterial pathogens and are all less effective than ampicillin. Against fungal pathogen C. albicans, compound 6d found better activity where as, 6a, 6c, 6g, 7b-c, 7e, 7g, 8b, 8d, 8h, 9b, 9d, 9f and $9 \mathrm{~g}$ were found to be equipotent compared to griseofulvin. None of the tested compounds found to be potent against $A$. fumigatus compared to standard drugs.

\section{Conclusion}

A new series of substituted 4-pyrazolyl-4H-pyrazolopyran 6a-h, -benzopyran 7a-h and naphthopyran 8a-h, 9a-h derivatives has been synthesized via an MCR approach and was characterized by elemental and spectral analysis. This synthetic strategy allows the construction of relatively complicated oxygen containing fused heterocyclic system as well as the introduction of various (hetero)aromatic substitutions into 4-position of pyran system. It can be concluded from antimicrobial screening (Table 1), against panel of human pathogens, that most of the synthesized naphthopyran derivatives $\mathbf{8 a - h}, \mathbf{9 a - h}$ was found to be highly active, compared to pyrazolopyran 6a-h and benzopyran 7a-h, against bacterial pathogens. Among them, compounds $\mathbf{8 b}, 8 \mathrm{~g}, 9 \mathrm{a}, 9 \mathrm{f}$ and $9 \mathrm{~h}$ were found to be the most active against the microorganisms employed for antibacterial activity. Antifungal activity of the compounds shows that most of the compounds found to be potent against $C$. albicans compared to A. fumigatus. It is worth mentioning that minor change in molecular configuration of these compounds profoundly influences the activity.

\section{Experimental Section}

General. All the reagents were obtained commercially and used with further purification. Solvents used were of analytical grade. All melting points were taken in open capillaries and are uncorrected. Thin-layer chromatography (TLC, on aluminium plates coated with silica gel $60 \mathrm{~F}_{254}, 0.25 \mathrm{~mm}$ thickness, Merck) was used for monitoring the progress of all reactions, purity and homogeneity of the synthesized compounds; eluent-toluene:ethyl acetate::7:3. UV radiation and iodine were used as the visualizing agents. Elemental analysis $(\% \mathrm{C}, \mathrm{H}, \mathrm{N})$ was carried out by Perkin-Elmer 2400 series-II elemental analyzer at Sophisticated Instrumentation Centre for Applied Research \& Training (SICART), Vallabh Vidyanagar and all compounds are within 
$\pm 0.4 \%$ of theory specified. The IR spectra were recorded in $\mathrm{KBr}$ on a Perkin-Elmer Spectrum GX FT-IR Spectrophotometer and only the characteristic peaks are reported in $\mathrm{cm}^{-1} .{ }^{1} \mathrm{H}$ NMR and ${ }^{13} \mathrm{C}$ NMR spectra were recorded in DMSO- $d_{6}$ on a Bruker Avance $400 \mathrm{~F}$ (MHz) spectrometer using solvent peak as internal standard at $400 \mathrm{MHz}$ and $100 \mathrm{MHz}$ respectively. Chemical shifts are reported in parts per million (ppm). Mass spectra were scanned on a Shimadzu LCMS 2010 spectrometer. 1-Phenyl-3-(het)aryl-pyrazole-4-carbaldehydes 1a-h were prepared by VilsmeierHaack reaction of acetophenone (het)arylhydrazones (Scheme 1). ${ }^{20}$

General procedure for the synthesis of 6-amino-4-(3-aryl-1-phenyl-1H-pyrazol-4-yl)-3methyl-2,4-dihydropyrano[2,3-c]pyrazole-5-carbonitrile 6a-h

3-Aryl-1-phenylpyrazole-4-carbaldehyde 1a-h (30 mmol), malononitrile 2 (30 mmol), 3-methyl2-pyrazolin-5-one 3 (30 mmol), ethanol $(15 \mathrm{~mL})$ and 3 drops of piperidine were charged in $100 \mathrm{~mL}$ round bottom flask with mechanical stirrer and condenser. The reaction mixture was slowly heated and refluxed for 3-4 hr. On completion of reaction, monitored by TLC (ethyl acetate:toluene::3:7), the reaction mixture was cooled to room temperature and the solid separated was filtered and washed with mixture of chloroform and methanol to obtain the pure compounds 6a-h. Analytical and spectroscopic characterization data of the synthesized compounds $\mathbf{6 a - h}$ are given below:

6-Amino-4-(1,3-diphenyl-1 $H$-pyrazol-4-yl)-3-methyl-2,4-dihydropyrano[2,3-c]pyrazole-5carbonitrile 6a. Yield: 60 \%. m.p. $209-211{ }^{\circ} \mathrm{C}$. Anal.Calcd. for $\mathrm{C}_{23} \mathrm{H}_{18} \mathrm{~N}_{6} \mathrm{O}$ (394.44 gm/mole): C 70.04, H 4.60, N 21.31 \%. Found: C 69.87, H 4.42, N 21.54 \%. IR (KBr, v, cm ${ }^{-1}$ ): 3410 \& 3385, 3270 (asym. \& sym. str. of $-\mathrm{NH}_{2}$ and $-\mathrm{NH}-$ str.), 2210 (-C $\equiv \mathrm{N}$ str.), 1220 (asym. str. of cyclic ArC-O-C ether). ${ }^{1} \mathrm{H}$ NMR (400 MHz, DMSO- $\left.d_{6}\right) \delta_{\mathrm{H}}(\mathrm{ppm}): 1.79$ (s, 3H, $\left.\mathrm{CH}_{3}\right), 4.59$ (s, 1H, pyran H4), 6.91 (s, 2H, $\mathrm{D}_{2} \mathrm{O}$ exch., $\mathrm{NH}_{2}$ ), 6.98-7.96 (m, 10H, Ar-H), 8.31 (s, 1H, pyrazole H5), 12.18 (s, $1 \mathrm{H}, \mathrm{D}_{2} \mathrm{O}$ exch., NH). ${ }^{13} \mathrm{C} \mathrm{NMR}\left(100 \mathrm{MHz}, \mathrm{DMSO}-d_{6}\right) \delta_{\mathrm{C}}(\mathrm{ppm}): 10.4\left(\mathrm{CH}_{3}\right), 27.2$ (pyran C4), 57.8 (pyran C3), 98.4 (pyran C5), $120.9(\mathrm{CN}), 114.5,117.3,124.8,126.5,127.3,128.9,129.5$, 129.7, 131.3, 135.8, 140.1, 151.2 (Ar-C), 154.7 (pyran C6), 161.6 (pyran C2).

6-Amino-4-(3-(4-bromophenyl)-1-phenyl-1 H-pyrazol-4-yl)-3-methyl-2,4-dihydropyrano[2,3-c]pyrazole-5-carbonitrile 6b. Yield: $58 \%$ m.p. $235-237{ }^{\circ} \mathrm{C}$. Anal.Calcd. for $\mathrm{C}_{23} \mathrm{H}_{17} \mathrm{BrN}_{6} \mathrm{O}$ (473.33 gm/mole): C 58.36, H 3.62, N 17.76 \%. Found: C 58.57, H 3.85, N 17.59 \%. IR (KBr, v, $\mathrm{cm}^{-1}$ ): $3395 \& 3360,3285$ (asym. \& sym. str. of $-\mathrm{NH}_{2}$ and $-\mathrm{NH}-\mathrm{str}$.), 2190 (-C $\equiv \mathrm{N}$ str.), 1235 (asym. str. of cyclic ArC-O-C ether). ${ }^{1} \mathrm{H}$ NMR (400 MHz, DMSO- $\left.d_{6}\right) \delta_{\mathrm{H}}(\mathrm{ppm}): 1.75$ (s, 3H, $\left.\mathrm{CH}_{3}\right), 4.78$ (s, 1H, pyran H4), 6.80 (s, 2H, D $\mathrm{O}_{2}$ exch., $\mathrm{NH}_{2}$ ), 7.01-8.08 (m, 9H, Ar-H), 8.39 (s, $1 \mathrm{H}$, pyrazole H5), 11.97 (s, $1 \mathrm{H}, \mathrm{D}_{2} \mathrm{O}$ exch., NH). ${ }^{13} \mathrm{C} \mathrm{NMR}\left(100 \mathrm{MHz}, \mathrm{DMSO}-d_{6}\right) \delta_{\mathrm{C}}(\mathrm{ppm})$ : $10.3\left(\mathrm{CH}_{3}\right.$ ), 26.6 (pyran C4), 58.6 (pyran C3), 97.7 (pyran C5), $121.3(\mathrm{CN}), 114.3,119.1,123.3$, 126.6, 126.9, 128.0, 129.4, 129.8, 131.2, 135.6, 140.0, 150.5 (Ar-C), 154.6 (pyran C6), 162.0 (pyran C2).

6-Amino-4-(3-(4-chlorophenyl)-1-phenyl-1H-pyrazol-4-yl)-3-methyl-2,4-dihydropyrano

[2,3-c]pyrazole-5-carbonitrile 6c. Yield: $61 \%$. m.p. 203-205 ${ }^{\circ} \mathrm{C}$. Anal.Calcd. for $\mathrm{C}_{23} \mathrm{H}_{17} \mathrm{ClN}_{6} \mathrm{O}$ (428.88 gm/mole): C 64.41, H 4.00, N 19.60 \%. Found: C 64.25, H 3.77, N 19.82 \%. IR (KBr, v, 
$\mathrm{cm}^{-1}$ ): $3410 \& 3355,3250$ (asym. \& sym. str. of $-\mathrm{NH}_{2}$ and $-\mathrm{NH}-\mathrm{str}$.), 2200 (-C $\equiv \mathrm{N}$ str.), 1210 (asym. str. of cyclic ArC-O-C ether). ${ }^{1} \mathrm{H}$ NMR (400 MHz, DMSO- $\left.d_{6}\right) \delta_{\mathrm{H}}(\mathrm{ppm}): 1.69$ (s, 3H, $\mathrm{CH}_{3}$ ), 4.60 (s, 1H, pyran H4), 6.94 (s, 2H, $\mathrm{D}_{2} \mathrm{O}$ exch., $\mathrm{NH}_{2}$ ), 6.99-7.94 (m, 9H, Ar-H), 8.35 (s, $1 \mathrm{H}$, pyrazole H5), 12.36 (s, $1 \mathrm{H}, \mathrm{D}_{2} \mathrm{O}$ exch., NH). ${ }^{13} \mathrm{C}$ NMR $\left(100 \mathrm{MHz}, \mathrm{DMSO}-d_{6}\right) \delta_{\mathrm{C}}(\mathrm{ppm})$ : $10.2\left(\mathrm{CH}_{3}\right), 26.7$ (pyran C4), 57.9 (pyran C3), 97.9 (pyran C5), $121.4(\mathrm{CN}), 113.6,118.9,125.0$, 126.4, 127.4, 128.6, 129.3, 130.0, 130.4, 136.2, 140.1, 150.6 (Ar-C), 156.1 (pyran C6), 161.3 (pyran C2).

6-Amino-4-(3-(4-fluorophenyl)-1-phenyl-1H-pyrazol-4-yl)-3-methyl-2,4-dihydropyrano[2,3c]pyrazole-5-carbonitrile 6d. Yield: 50 \%. m.p. 229-231 ${ }^{\circ} \mathrm{C}$. Anal.Calcd. for $\mathrm{C}_{23} \mathrm{H}_{17} \mathrm{FN}_{6} \mathrm{O}$ (412.43 gm/mole): C 66.98, H 4.16, N 20.38 \%. Found: C 66.69, H 4.39, N 20.23 \%. IR (KBr, v, $\mathrm{cm}^{-1}$ ): $3400 \& 3370,3285$ (asym. \& sym. str. of $-\mathrm{NH}_{2}$ and $-\mathrm{NH}-$ str.), 2190 (-C $\equiv \mathrm{N}$ str.), 1255 (asym. str. of cyclic ArC-O-C ether). ${ }^{1} \mathrm{H}$ NMR (400 MHz, DMSO- $\left.d_{6}\right) \delta_{\mathrm{H}}(\mathrm{ppm}): 1.80(\mathrm{~s}, 3 \mathrm{H}$, $\mathrm{CH}_{3}$ ), 4.88 (s, $1 \mathrm{H}$, pyran H4), 6.79 (s, 2H, $\mathrm{D}_{2} \mathrm{O}$ exch., $\mathrm{NH}_{2}$ ), 6.87-7.89 (m, 9H, Ar-H), 8.27 (s, $1 \mathrm{H}$, pyrazole H5), 12.54 (s, 1H, $\mathrm{D}_{2} \mathrm{O}$ exch., NH). ${ }^{13} \mathrm{C}$ NMR (100 MHz, DMSO- $\left.d_{6}\right) \delta_{\mathrm{C}}(\mathrm{ppm})$ : $11.4\left(\mathrm{CH}_{3}\right.$ ), 27.4 (pyran C4), 58.5 (pyran C3), 98.3 (pyran C5), $120.9(\mathrm{CN}), 115.0,117.4,124.4$, 126.2, 127.3, 128.8, 129.7, 130.1, 130.5, 135.5, 139.2, 150.9 (Ar-C), 155.4 (pyran C6), 160.8 (pyran C2).

6-Amino-4-(3-(4-methoxyphenyl)-1-phenyl-1H-pyrazol-4-yl)-3-methyl-2,4-dihydropyrano [2,3-c]pyrazole-5-carbonitrile 6e. Yield: $60 \%$ m.p. 215-217 ${ }^{\circ} \mathrm{C}$. Anal.Calcd. for $\mathrm{C}_{24} \mathrm{H}_{20} \mathrm{~N}_{6} \mathrm{O}_{2}$ (424.47 gm/mole): C 67.91, H 4.75, N 19.80 \%. Found: C 67.79, H 4.49, N 20.07 \%. IR (KBr, v, $\mathrm{cm}^{-1}$ ): $3405 \&$ 3360, 3255 (asym. \& sym. str. of $-\mathrm{NH}_{2}$ and $-\mathrm{NH}-$ str.), 2205 (-C $\equiv \mathrm{N}$ str.), 1230 (asym. str. of cyclic ArC-O-C ether). ${ }^{1} \mathrm{H}$ NMR (400 MHz, DMSO- $\left.d_{6}\right) \delta_{\mathrm{H}}$ (ppm): 1.75 (s, 3H, $\left.\mathrm{CH}_{3}\right), 3.79$ (s, 3H, $\left.\mathrm{OCH}_{3}\right), 4.85$ (s, 1H, pyran $\left.\mathrm{H} 4\right), 6.82$ (s, 2H, $\mathrm{D}_{2} \mathrm{O}$ exch., $\left.\mathrm{NH}_{2}\right)$, 6.96-7.91 (m, $9 \mathrm{H}, \mathrm{Ar}-\mathrm{H}), 8.45$ (s, 1H, pyrazole H5), 12.01 (s, 1H, D $\mathrm{D}_{2} \mathrm{O}$ exch., NH). ${ }^{13} \mathrm{C} \mathrm{NMR}(100 \mathrm{MHz}$, DMSO- $\left.d_{6}\right) \delta_{\mathrm{C}}(\mathrm{ppm}): 10.2\left(\mathrm{CH}_{3}\right), 26.7$ (pyran $\left.\mathrm{C} 4\right), 55.5\left(\mathrm{OCH}_{3}\right), 57.4$ (pyran $\left.\mathrm{C} 3\right), 97.9$ (pyran C5), 121.4 (CN), 114.1, 118.1, 124.5, 126.0, 126.4, 128.4, 129.9, 131.6, 135.9, 139.8, 151.3 (ArC), 154.9 (pyran C6), $159.4\left(\right.$ ArC- $\left.-\mathrm{OCH}_{3}\right), 161.1$ (pyran C2). MS: $425.1\left(\mathrm{M}^{+}+1\right)$.

6-Amino-3-methyl-4-(1-phenyl-3-(4-methylphenyl)-1H-pyrazol-4-yl)-2,4-dihydropyrano [2,3-c]pyrazole-5-carbonitrile 6f. Yield: $58 \%$ m.p. 208-210 ${ }^{\circ} \mathrm{C}$. Anal.Calcd. for $\mathrm{C}_{24} \mathrm{H}_{20} \mathrm{~N}_{6} \mathrm{O}$ (408.47 gm/mole): C 70.57, H 4.94, N 20.58 \%. Found: C 70.34, H 5.17, N 20.31 \%. IR (KBr, v, $\mathrm{cm}^{-1}$ ): $3385 \&$ 3345, 3270 (asym. \& sym. str. of $-\mathrm{NH}_{2}$ and $-\mathrm{NH}-$ str.), 2195 (-C $\equiv \mathrm{N}$ str.), 1250 (asym. str. of cyclic ArC-O-C ether). ${ }^{1} \mathrm{H}$ NMR (400 MHz, DMSO- $\left.d_{6}\right) \delta_{\mathrm{H}}(\mathrm{ppm}): 1.79$ (s, 3H, $\left.\mathrm{CH}_{3}\right), 2.34$ (s, 3H, tolyl-CH 3$), 4.61$ (s, $1 \mathrm{H}$, pyran $\left.\mathrm{H} 4\right), 6.87$ (s, 2H, $\mathrm{D}_{2} \mathrm{O}$ exch., $\left.\mathrm{NH}_{2}\right), 7.06-8.14$ (m, 9H, Ar-H), 8.32 (s, 1H, pyrazole H5), 12.15 (s, 1H, $\mathrm{D}_{2} \mathrm{O}$ exch., NH). ${ }^{13} \mathrm{C} \mathrm{NMR}(100 \mathrm{MHz}$, DMSO- $\left.d_{6}\right) \delta_{\mathrm{C}}$ (ppm): $10.5\left(\mathrm{CH}_{3}\right), 20.9\left(\right.$ tolyl- $\left.\mathrm{CH}_{3}\right), 27.3$ (pyran $\left.\mathrm{C} 4\right), 57.8$ (pyran C3), 98.2 (pyran C5), $120.8(\mathrm{CN}), 113.2,118.4,125.1,126.8,126.6,128.1,129.3,129.9,130.8,136.1$, 140.0, 150.8 (Ar-C), 154.8 (pyran C6), 161.4 (pyran C2).

6-Amino-3-methyl-4-(3-(4-nitrophenyl)-1-phenyl-1 $H$-pyrazol-4-yl)-2,4-dihydropyrano[2,3c]pyrazole-5-carbonitrile 6g. Yield: 55 \%. m.p.190-192 ${ }^{\circ} \mathrm{C}$. Anal.Calcd. for $\mathrm{C}_{23} \mathrm{H}_{17} \mathrm{~N}_{7} \mathrm{O}_{3}$ (439.44 gm/mole): C 62.87, H 3.90, N 22.31 \%. Found: C 63.05, H 3.74, N 22.09 \%. IR (KBr, v, 
$\mathrm{cm}^{-1}$ ): $3395 \&$ 3350, 3195 (asym. \& sym. str. of $-\mathrm{NH}_{2}$ and $-\mathrm{NH}-$ str.), 2185 (-C $\equiv \mathrm{N}$ str.), 1245 (asym. str. of cyclic ArC-O-C ether). ${ }^{1} \mathrm{H}$ NMR (400 MHz, DMSO- $\left.d_{6}\right) \delta_{\mathrm{H}}(\mathrm{ppm}): 1.76$ (s, 3H, $\left.\mathrm{CH}_{3}\right), 4.76$ (s, 1H, pyran H4), 6.86 (s, 2H, $\mathrm{D}_{2} \mathrm{O}$ exch., $\mathrm{NH}_{2}$ ), 6.97-8.22 (m, 9H, Ar-H), 8.41 (s, $1 \mathrm{H}$, pyrazole H5), 12.40 (s, $1 \mathrm{H}, \mathrm{D}_{2} \mathrm{O}$ exch., NH). ${ }^{13} \mathrm{C}$ NMR $\left(100 \mathrm{MHz}, \mathrm{DMSO}-d_{6}\right) \delta_{\mathrm{C}}(\mathrm{ppm})$ : $11.3\left(\mathrm{CH}_{3}\right), 26.8$ (pyran C4), 59.0 (pyran C3), 97.9 (pyran C5), $120.8(\mathrm{CN}), 114.8,117.8,124.1$, 126.1, 127.4, 128.9, 129.6, 129.7, 131.6, 136.2, 140.2, 151.1 (Ar-C), 156.1 (pyran C6), 160.9 (pyran C2).

6-Amino-3-methyl-4-(1-phenyl-3-(thien-2-yl)-1 H-pyrazol-4-yl)-2,4-dihydropyrano[2,3c]pyrazole-5-carbonitrile 6h. Yield: 57 \%. m.p. 230-232 ${ }^{\circ} \mathrm{C}$. Anal.Calcd. for $\mathrm{C}_{21} \mathrm{H}_{16} \mathrm{~N}_{6} \mathrm{OS}$ (400.47 gm/mole): C 62.99, H 4.03, N 20.99 \% Found: C 63.29, H 4.32, N 20.64 \%. IR (KBr, v, $\mathrm{cm}^{-1}$ ): $3415 \& 3380,3200$ (asym. \& sym. str. of $-\mathrm{NH}_{2}$ and $-\mathrm{NH}-$ str.), 2190 (-C $\equiv \mathrm{N}$ str.), 1220 (asym. str. of cyclic ArC-O-C ether). ${ }^{1} \mathrm{H}$ NMR (400 MHz, DMSO- $\left.d_{6}\right) \delta_{\mathrm{H}}(\mathrm{ppm}): 1.72$ (s, 3H, $\mathrm{CH}_{3}$ ), 4.59 (s, $1 \mathrm{H}$, pyran H4), 6.83 (s, 2H, $\mathrm{D}_{2} \mathrm{O}$ exch., $\mathrm{NH}_{2}$ ), 7.03-8.09 (m, 8H, Ar-H), 8.35 (s, $1 \mathrm{H}$, pyrazole H5), 12.28 (s, 1H, $\mathrm{D}_{2} \mathrm{O}$ exch., NH). ${ }^{13} \mathrm{C}$ NMR (100 MHz, DMSO- $\left.d_{6}\right) \delta_{\mathrm{C}}(\mathrm{ppm})$ : $10.2\left(\mathrm{CH}_{3}\right), 26.8$ (pyran C4), 58.4 (pyran C3), 97.8 (pyran C5), $121.0(\mathrm{CN}), 114.9,119.0,124.4$, 126.0, 126.3, 128.7, 129.7, 130.0, 131.9, 136.2, 139.9, 151.0 (Ar-C), 155.6 (pyran C6), 162.0 (pyran C2).

General procedure for the synthesis of 2-amino-4-(3-aryl-1-phenyl-1H-pyrazol-4-yl)-7,7dimethyl-5-oxo-5,6,7,8-tetrahydro-4H-chromene-3-carbonitrile 7a-h

3-Aryl-1-phenylpyrazole-4-carbaldehyde 1a-h (30 mmol), malononitrile 2 (30 mmol), 5,5dimethyl-1,3-cyclohexanedione (dimedone) 4 (30 mmol), ethanol $(15 \mathrm{~mL})$ and 3 drops of piperidine were charged in $100 \mathrm{~mL}$ round bottom flask with mechanical stirrer and condenser. The reaction mixture was slowly heated and refluxed for 3-4 hr. On completion of reaction, monitored by TLC (ethyl acetate:toluene::3:7), the reaction mixture was cooled to room temperature and the solid separated was filtered, washed with mixture of chloroform and methanol and dried to obtain the pure compounds 7a-h. Analytical and spectroscopic characterization data of the synthesized compounds $\mathbf{7 a - h}$ are given below:

\section{2-Amino-4-(1,3-diphenyl-1H-pyrazol-4-yl)-7,7-dimethyl-5-oxo-5,6,7,8-tetrahydro-4H-}

chromene-3-carbonitrile 7a. Yield: 69 \%. m.p. 209-211 ${ }^{\circ} \mathrm{C}$. Anal.Calcd. for $\mathrm{C}_{27} \mathrm{H}_{24} \mathrm{~N}_{4} \mathrm{O}_{2}$ (436.52 gm/mole): C 74.29, H 5.54, N 12.84 \%. Found: C 74.13, H 5.38, N 13.01 \%. IR (KBr, v, $\mathrm{cm}^{-1}$ ): $3430 \& 3300$ (asym. \& sym. str. of $\left.-\mathrm{NH}_{2}\right), 2210(-\mathrm{C} \equiv \mathrm{N}$ str.), $1680(\mathrm{C}=\mathrm{O}$ str. of cyclic ketone), 1235 (asym. str. of cyclic ArC-O-C ether). ${ }^{1} \mathrm{H}$ NMR (400 MHz, DMSO- $\left.d_{6}\right) \delta_{\mathrm{H}}(\mathrm{ppm})$ : $1.04\left(\mathrm{~s}, 3 \mathrm{H}, \mathrm{CH}_{3}\right), 1.11\left(\mathrm{~s}, 3 \mathrm{H}, \mathrm{CH}_{3}\right), 2.19-2.47\left(4 \mathrm{H}, \mathrm{m}, 2 \times \mathrm{CH}_{2}\right), 4.43(\mathrm{~s}, 1 \mathrm{H}$, pyran $\mathrm{H} 4), 6.88$ (s, $2 \mathrm{H}, \mathrm{D}_{2} \mathrm{O}$ exch., $\left.\mathrm{NH}_{2}\right), 6.96-7.91(\mathrm{~m}, 10 \mathrm{H}, \mathrm{Ar}-\mathrm{H}), 8.31\left(\mathrm{~s}, 1 \mathrm{H}\right.$, pyrazole H5). ${ }^{13} \mathrm{C}$ NMR $(100$ MHz, DMSO-d $\left.d_{6}\right) \delta_{\mathrm{C}}(\mathrm{ppm}): 25.6\left(\mathrm{CH}_{3}\right), 27.9\left(\mathrm{CH}_{3}\right), 28.5$ (pyran $\left.\mathrm{C} 4\right), 32.5\left(\underline{\mathrm{C}}\left(\mathrm{CH}_{3}\right)_{2}\right), 42.1$ $\left(\mathrm{CH}_{2}\right), 51.2\left(\underline{\mathrm{CH}}_{2}-\mathrm{CO}\right), 58.0$ (pyran $\mathrm{C} 3$ ), 112.7 (pyran $\left.\mathrm{C} 5\right), 121.8(\mathrm{CN}), 114.3,118.0,125.3$, 125.8, 126.9, 127.8, 128.8, 131.0, 136.1, 138.8, 151.1 (Ar-C), 157.9 (pyran C6), 163.1 (pyran $\mathrm{C} 2), 196.1(\mathrm{C}=\mathrm{O})$. 
2-Amino-4-(3-(4-bromophenyl)-1-phenyl-1 H-pyrazol-4-yl)-7,7-dimethyl-5-oxo-5,6,7,8tetrahydro-4H-chromene-3-carbonitrile 7b. Yield: $60 \%$ m.p. 227-229 ${ }^{\circ} \mathrm{C}$. Anal.Calcd. for $\mathrm{C}_{27} \mathrm{H}_{23} \mathrm{BrN}_{4} \mathrm{O}_{2}$ (515.41 gm/mole): C 62.92, H 4.50, N 10.87 \%. Found: C 62.78, H 4.66, N 10.71 \%. IR (KBr, v, cm $\left.{ }^{-1}\right): 3420$ \& 3295 (asym. \& sym. str. of $\left.-\mathrm{NH}_{2}\right), 2210(-\mathrm{C} \equiv \mathrm{N}$ str. $), 1685(\mathrm{C}=\mathrm{O}$ str. of cyclic ketone), 1255 (asym. str. of cyclic ArC-O-C ether). ${ }^{1} \mathrm{H}$ NMR (400 MHz, DMSO- $d_{6}$ ) $\delta_{\mathrm{H}}(\mathrm{ppm}): 0.97\left(\mathrm{~s}, 3 \mathrm{H}, \mathrm{CH}_{3}\right), 1.06\left(\mathrm{~s}, 3 \mathrm{H}, \mathrm{CH}_{3}\right), 2.15-2.50\left(4 \mathrm{H}, \mathrm{m}, 2 \times \mathrm{CH}_{2}\right), 4.57(\mathrm{~s}, 1 \mathrm{H}$, pyran H4), 6.85 (s, 2H, $\mathrm{D}_{2} \mathrm{O}$ exch., $\mathrm{NH}_{2}$ ), $7.01-7.93$ (m, 10H, Ar-H), 8.39 (s, 1H, pyrazole H5). ${ }^{13} \mathrm{C}$ NMR (100 MHz, DMSO- $\left.d_{6}\right) \delta_{\mathrm{C}}(\mathrm{ppm}): 25.8\left(\mathrm{CH}_{3}\right), 27.7\left(\mathrm{CH}_{3}\right), 28.6$ (pyran $\left.\mathrm{C} 4\right), 33.0\left(\underline{\mathrm{C}}\left(\mathrm{CH}_{3}\right)_{2}\right)$, $41.8\left(\mathrm{CH}_{2}\right), 52.0\left(\underline{\mathrm{CH}}_{2}-\mathrm{CO}\right), 57.7$ (pyran $\mathrm{C} 3$ ), 113.9 (pyran $\left.\mathrm{C} 5\right), 120.3(\mathrm{CN}), 115.0,117.8,124.9$, 125.4, 126.8, 128.2, 129.2, 130.9, 136.2, 139.1, 150.9 (Ar-C), 157.4 (pyran C6), 162.8 (pyran $\mathrm{C} 2), 194.9(\mathrm{C}=\mathrm{O})$.

2-Amino-4-(3-(4-chlorophenyl)-1-phenyl-1 $H$-pyrazol-4-yl)-7,7-dimethyl-5-oxo-5,6,7,8tetrahydro-4H-chromene-3-carbonitrile 7c. Yield: 63 \%. m.p. 229-231 ${ }^{\circ} \mathrm{C}$. Anal.Calcd. for $\mathrm{C}_{27} \mathrm{H}_{23} \mathrm{ClN}_{4} \mathrm{O}_{2}$ (470.96 gm/mole): C 68.86, H 4.92, N 11.90 \%. Found: C 69.03, H 4.73, N 11.75 \%. IR ( $\left.\mathrm{KBr}, v, \mathrm{~cm}^{-1}\right): 3455$ \& 3290 (asym. \& sym. str. of $\left.-\mathrm{NH}_{2}\right), 2200(-\mathrm{C} \equiv \mathrm{N}$ str.), $1680(\mathrm{C}=\mathrm{O}$ str. of cyclic ketone), 1230 (asym. str. of cyclic ArC-O-C ether). ${ }^{1} \mathrm{H}$ NMR (400 MHz, DMSO- $d_{6}$ ) $\delta_{\mathrm{H}}(\mathrm{ppm}): 1.02\left(\mathrm{~s}, 3 \mathrm{H}, \mathrm{CH}_{3}\right), 1.10\left(\mathrm{~s}, 3 \mathrm{H}, \mathrm{CH}_{3}\right), 2.16-2.49\left(4 \mathrm{H}, \mathrm{m}, 2 \times \mathrm{CH}_{2}\right), 4.47(\mathrm{~s}, 1 \mathrm{H}$, pyran 4H), 6.93 (s, 2H, D $2 \mathrm{O}$ exch., $\mathrm{NH}_{2}$ ), 7.04-7.90 (m, 10H, Ar-H), 8.32 (s, 1H, pyrazole H5). ${ }^{13} \mathrm{C}$ NMR (100 MHz, DMSO- $\left.d_{6}\right) \delta_{\mathrm{C}}(\mathrm{ppm}): 25.7\left(\mathrm{CH}_{3}\right), 27.9\left(\mathrm{CH}_{3}\right), 28.4$ (pyran C4), $31.9\left(\underline{\mathrm{C}}\left(\mathrm{CH}_{3}\right)_{2}\right)$, $40.6\left(\mathrm{CH}_{2}\right), 50.8\left(\underline{\mathrm{CH}}_{2}-\mathrm{CO}\right), 57.3$ (pyran $\mathrm{C} 3$ ), 115.0 (pyran $\left.\mathrm{C} 5\right), 121.5(\mathrm{CN}), 114.9,118.3,125.7$, 126.0, 127.0, 128.6, 129.1, 130.6, 136.5, 139.3, 152.1 (Ar-C), 158.1 (pyran C6), 163.2 (pyran $\mathrm{C} 2), 195.7(\mathrm{C}=\mathrm{O})$.

2-Amino-4-(3-(4-fluorophenyl)-1-phenyl-1H-pyrazol-4-yl)-7,7-dimethyl-5-oxo-5,6,7,8tetrahydro-4H-chromene-3-carbonitrile 7d. Yield: $65 \%$ m.p. 222-224 ${ }^{\circ} \mathrm{C}$. Anal.Calcd. for $\mathrm{C}_{27} \mathrm{H}_{23} \mathrm{~N}_{4} \mathrm{FO}_{2}$ (454.51 gm/mole): C 71.35, H 5.10, N 12.33 \%. Found: C 71.21, H 4.94, N 12.45 \%. IR ( $\left.\mathrm{KBr}, v, \mathrm{~cm}^{-1}\right): 3435 \& 3350$ (asym. \& sym. str. of $\left.-\mathrm{NH}_{2}\right), 2190(-\mathrm{C} \equiv \mathrm{N}$ str.), $1690(\mathrm{C}=\mathrm{O}$ str. of cyclic ketone), 1240 (asym. str. of cyclic ArC-O-C ether). ${ }^{1} \mathrm{H}$ NMR (400 MHz, DMSO- $d_{6}$ ) $\delta_{\mathrm{H}}(\mathrm{ppm}): 0.94\left(\mathrm{~s}, 3 \mathrm{H}, \mathrm{CH}_{3}\right), 1.03\left(\mathrm{~s}, 3 \mathrm{H}, \mathrm{CH}_{3}\right), 2.09-2.48\left(4 \mathrm{H}, \mathrm{m}, 2 \times \mathrm{CH}_{2}\right), 4.55(\mathrm{~s}, 1 \mathrm{H}$, pyran $\mathrm{H} 4), 6.84$ (s, 2H, D $2 \mathrm{O}$ exch., $\left.\mathrm{NH}_{2}\right), 7.20-7.90(\mathrm{~m}, 10 \mathrm{H}, \mathrm{Ar}-\mathrm{H}), 8.27\left(\mathrm{~s}, 1 \mathrm{H}\right.$, pyrazole H5). ${ }^{13} \mathrm{C}$ NMR (100 MHz, DMSO- $\left.d_{6}\right) \delta_{\mathrm{C}}(\mathrm{ppm}): 25.6\left(\mathrm{CH}_{3}\right), 27.6\left(\mathrm{CH}_{3}\right), 28.6$ (pyran $\left.\mathrm{C} 4\right), 32.4\left(\underline{\mathrm{C}}\left(\mathrm{CH}_{3}\right)_{2}\right)$, $42.0\left(\mathrm{CH}_{2}\right), 51.5\left(\underline{\mathrm{CH}}_{2}-\mathrm{CO}\right), 59.4$ (pyran $\left.\mathrm{C} 3\right), 114.4$ (pyran $\left.\mathrm{C} 5\right), 120.8(\mathrm{CN}), 115.1,118.5,126.2$, 126.2, 127.2, 127.4, 128.6, 131.1, 136.2, 138.6, 150.9 (Ar-C), 157.9 (pyran C6), 162.7 (pyran $\mathrm{C} 2), 194.8(\mathrm{C}=\mathrm{O})$.

2-Amino-4-(3-(4-methoxyphenyl)-1-phenyl-1 $H$-pyrazol-4-yl)-7,7-dimethyl-5-oxo-5,6,7,8tetrahydro-4H-chromene-3-carbonitrile 7e. Yield: $58 \%$ m.p. $216-217{ }^{\circ} \mathrm{C}$. Anal.Calcd. for $\mathrm{C}_{28} \mathrm{H}_{26} \mathrm{~N}_{4} \mathrm{O}_{3}$ (466.54 gm/mole): C 72.09, H 5.62, N 12.01 \%. Found: C 71.91, H 5.88, N 11.84 \%. IR ( $\left.\mathrm{KBr}, v, \mathrm{~cm}^{-1}\right): 3400 \& 3285$ (asym. \& sym. str. of $\left.-\mathrm{NH}_{2}\right), 2195(-\mathrm{C} \equiv \mathrm{N}$ str.), $1700(\mathrm{C}=\mathrm{O}$ str. of cyclic ketone), 1235 (asym. str. of cyclic ArC-O-C ether). ${ }^{1} \mathrm{H}$ NMR (400 MHz, DMSO- $d_{6}$ ) $\delta_{\mathrm{H}}(\mathrm{ppm}): 0.97\left(\mathrm{~s}, 3 \mathrm{H}, \mathrm{CH}_{3}\right), 1.01\left(\mathrm{~s}, 3 \mathrm{H}, \mathrm{CH}_{3}\right), 2.11-2.43\left(4 \mathrm{H}, \mathrm{m}, 2 \times \mathrm{CH}_{2}\right), 3.82\left(\mathrm{~s}, 3 \mathrm{H}, \mathrm{OCH}_{3}\right)$, 4.42 (s, 1H, pyran H4), 6.99 (s, 2H, D $2 \mathrm{O}$ exch., $\mathrm{NH}_{2}$ ), 7.03-7.85 (m, 10H, Ar-H), 8.37 (s, 1H, 
pyrazole H5). ${ }^{13} \mathrm{C}$ NMR (100 MHz, DMSO- $\left.d_{6}\right) \delta_{\mathrm{C}}(\mathrm{ppm}): 25.8\left(\mathrm{CH}_{3}\right), 27.9\left(\mathrm{CH}_{3}\right), 28.4$ (pyran C4), $32.1\left(\underline{\mathrm{C}}\left(\mathrm{CH}_{3}\right)_{2}\right), 40.2\left(\mathrm{CH}_{2}\right), 50.6\left(\mathrm{CH}_{2}-\mathrm{CO}\right), 55.6\left(\mathrm{OCH}_{3}\right), 59.0$ (pyran $\left.\mathrm{C} 3\right), 112.3$ (pyran C5), $120.3(\mathrm{CN}), 114.1,118.2,126.3,126.6,127.8,129.8,130.2$, 136.5, 139.9, 151.2 (Ar-C), 157.5 (pyran C6), $159.4\left(\mathrm{Ar} \underline{\mathrm{C}}-\mathrm{OCH}_{3}\right), 162.3$ (pyran C2), $196.4(\mathrm{C}=\mathrm{O})$. MS: $467.2\left(\mathrm{M}^{+}+1\right)$.

2-Amino-7,7-dimethyl-5-oxo-4-(1-phenyl-3-(4-methylphenyl)-1H-pyrazol-4-yl)-5,6,7,8tetrahydro-4H-chromene-3-carbonitrile 7f. Yield: $61 \%$ m.p. 207-209 ${ }^{\circ} \mathrm{C}$. Anal.Calcd. for $\mathrm{C}_{28} \mathrm{H}_{26} \mathrm{~N}_{4} \mathrm{O}_{2}$ (450.54 gm/mole): C 74.65, H 5.82, N $12.44 \%$. Found: C 74.76, H 5.95, N 12.19 \%. IR ( $\left.\mathrm{KBr}, v, \mathrm{~cm}^{-1}\right): 3425 \& 3335$ (asym. \& sym. str. of $\left.-\mathrm{NH}_{2}\right), 2205(-\mathrm{C} \equiv \mathrm{N}$ str.), $1695(\mathrm{C}=\mathrm{O}$ str. of cyclic ketone), 1250 (asym. str. of cyclic ArC-O-C ether). ${ }^{1} \mathrm{H}$ NMR (400 MHz, DMSO- $d_{6}$ ) $\delta_{\mathrm{H}}(\mathrm{ppm}): 1.02\left(\mathrm{~s}, 3 \mathrm{H}, \mathrm{CH}_{3}\right), 1.12\left(\mathrm{~s}, 3 \mathrm{H}, \mathrm{CH}_{3}\right), 2.17-2.45\left(4 \mathrm{H}, \mathrm{m}, 2 \times \mathrm{CH}_{2}\right), 2.37$ (s, 3H, tolyl$\mathrm{CH}_{3}$ ), 4.46 (s, 1H, pyran H4), 6.94 (s, 2H, $\mathrm{D}_{2} \mathrm{O}$ exch., $\mathrm{NH}_{2}$ ), 7.22-8.04 (m, 10H, Ar-H), 8.39 (pyrazole H5). ${ }^{13} \mathrm{C}$ NMR (100 MHz, DMSO- $\left.d_{6}\right) \delta_{\mathrm{C}}(\mathrm{ppm}): 21.1\left(\right.$ tolyl- $\left.\mathrm{CH}_{3}\right), 25.8\left(\mathrm{CH}_{3}\right), 27.7$ $\left(\mathrm{CH}_{3}\right), 28.5$ (pyran $\left.\mathrm{C} 4\right), 31.9\left(\underline{\mathrm{C}}\left(\mathrm{CH}_{3}\right)_{2}\right), 40.7\left(\mathrm{CH}_{2}\right), 52.1\left(\underline{\mathrm{CH}_{2}}-\mathrm{CO}\right), 56.5$ (pyran $\left.\mathrm{C} 3\right), 113.4$ (pyran C5), $120.6(\mathrm{CN}), 114.2,118.0,125.6,126.7,127.7,128.5,130.7,131.3,136.8,138.4$, 151.3, (Ar-C), 157.4 (pyran C6), 163.0 (pyran C2), $195.0(\mathrm{C}=\mathrm{O})$.

2-Amino-7,7-dimethyl-4-(3-(4-nitrophenyl)-1-phenyl-1 $H$-pyrazol-4-yl)-5-oxo-5,6,7,8tetrahydro-4H-chromene-3-carbonitrile 7g. Yield: $64 \%$ m.p. 224-226 ${ }^{\circ} \mathrm{C}$. Anal.Calcd. for $\mathrm{C}_{27} \mathrm{H}_{23} \mathrm{~N}_{5} \mathrm{O}_{4}$ (481.51 gm/mole): C 67.35, H 4.82, N 14.54 \%. Found: C 67.27, H 4.68, N 14.42 \%. IR ( $\left.\mathrm{KBr}, v, \mathrm{~cm}^{-1}\right): 3410 \& 3320$ (asym. \& sym. str. of $\left.-\mathrm{NH}_{2}\right), 2200(-\mathrm{C} \equiv \mathrm{N}$ str. $), 1690(\mathrm{C}=\mathrm{O}$ str. of cyclic ketone), 1245 (asym. str. of cyclic ArC-O-C ether). ${ }^{1} \mathrm{H}$ NMR (400 MHz, DMSO- $d_{6}$ ) $\delta_{\mathrm{H}}(\mathrm{ppm}): 0.96\left(\mathrm{~s}, 3 \mathrm{H}, \mathrm{CH}_{3}\right), 1.04\left(\mathrm{~s}, 3 \mathrm{H}, \mathrm{CH}_{3}\right), 2.10-2.51\left(4 \mathrm{H}, \mathrm{m}, 2 \times \mathrm{CH}_{2}\right), 4.52(\mathrm{~s}, 1 \mathrm{H}$, pyran H4), 6.90 (s, 2H, $\mathrm{D}_{2} \mathrm{O}$ exch., $\mathrm{NH}_{2}$ ), 7.29-8.01 (m, 10H, Ar-H), 8.29 (pyrazole H5). ${ }^{13} \mathrm{C}$ NMR $\left(100 \mathrm{MHz}, \mathrm{DMSO}-d_{6}\right) \delta_{\mathrm{C}}(\mathrm{ppm}): 25.7\left(\mathrm{CH}_{3}\right), 27.8\left(\mathrm{CH}_{3}\right), 28.5$ (pyran C4), $32.7\left(\underline{\mathrm{C}}\left(\mathrm{CH}_{3}\right)_{2}\right), 41.3$ $\left(\mathrm{CH}_{2}\right), 52.0\left(\underline{\mathrm{CH}}_{2}-\mathrm{CO}\right), 56.2$ (pyran $\mathrm{C} 3$ ), 113.5 (pyran $\left.\mathrm{C} 5\right), 121.1(\mathrm{CN}), 114.9,117.8,126.0$, $126.5,127.4,127.6,129.7,131.2,136.0,139.4,152.0$ (Ar-C), 158.0 (pyran C6), 162.9 (pyran $\mathrm{C} 2), 197.1(\mathrm{C}=\mathrm{O})$.

2-Amino-7,7-dimethyl-5-oxo-4-(1-phenyl-3-(thien-2-yl)-1 $H$-pyrazol-4-yl)-5,6,7,8-tetrahydro -4H-chromene-3-carbonitrile 7h. Yield: $66 \%$ m.p. 204-206 ${ }^{\circ} \mathrm{C}$. Anal.Calcd. for $\mathrm{C}_{25} \mathrm{H}_{22} \mathrm{~N}_{4} \mathrm{O}_{2} \mathrm{~S}$ (442.55 gm/mole): C 67.85, H 5.01, N 12.66 \%. Found: C 67.60, H 5.24, N 12.47 \%. IR (KBr, v, $\mathrm{cm}^{-1}$ ): $3430 \& 3345$ (asym. \& sym. str. of $\left.-\mathrm{NH}_{2}\right), 2195$ (-C $\equiv \mathrm{N}$ str.), $1685(\mathrm{C}=\mathrm{O}$ str. of cyclic ketone), 1240 (asym. str. of cyclic ArC-O-C ether). ${ }^{1} \mathrm{H}$ NMR (400 MHz, DMSO- $\left.d_{6}\right) \delta_{\mathrm{H}}(\mathrm{ppm})$ : $1.03\left(\mathrm{~s}, 3 \mathrm{H}, \mathrm{CH}_{3}\right), 1.12\left(\mathrm{~s}, 3 \mathrm{H}, \mathrm{CH}_{3}\right), 2.18-2.42\left(4 \mathrm{H}, \mathrm{m}, 2 \times \mathrm{CH}_{2}\right), 4.49$ (s, 1H, pyran H4), 6.87 (s, $2 \mathrm{H}, \mathrm{D}_{2} \mathrm{O}$ exch., $\left.\mathrm{NH}_{2}\right), 7.09-8.06(\mathrm{~m}, 10 \mathrm{H}, \mathrm{Ar}-\mathrm{H}), 8.41$ (pyrazole H5). ${ }^{13} \mathrm{C} \mathrm{NMR}(100 \mathrm{MHz}$, DMSO-d $\left.d_{6}\right) \delta_{\mathrm{C}}$ (ppm): $25.8\left(\mathrm{CH}_{3}\right), 27.7\left(\mathrm{CH}_{3}\right), 28.4$ (pyran C4), $32.3\left(\underline{\mathrm{C}}\left(\mathrm{CH}_{3}\right)_{2}\right), 42.0\left(\mathrm{CH}_{2}\right), 51.8$ $\left(\underline{\mathrm{CH}}_{2}-\mathrm{CO}\right), 58.0$ (pyran C3), 115.1 (pyran C5), $121.4(\mathrm{CN}), 114.2,118.2,126.1,126.3,127.1$, 128.3, 129.3, 130.9, 136.8, 139.2, 150.9 (Ar-C), 157.8 (pyran C6), 162.3 (pyran C2), 195.9 $(\mathrm{C}=\mathrm{O})$. 
General procedure for the synthesis of 2-amino-4-(3-aryl-1-phenyl-1H-pyrazol-4-yl)-4Hbenzo[ $h]$ chromene-3-carbonitrile 8a-h and 3-amino-1-(3-aryl-1-phenyl-1H-pyrazol-4-yl)$1 \boldsymbol{H}$-benzo[f]chromene-2-carbonitrile 9a-h

3-Aryl-1-phenylpyrazole-4-carbaldehyde 1a-h $(30 \mathrm{mmol})$, malononitrile 2 (30 mmol), 1- or 2naphthol 5 (30 mmol), acetonitrile $(\mathrm{MeCN})(15 \mathrm{~mL})$ and 3 drops of piperidine were charged in $100 \mathrm{~mL}$ round bottom flask with mechanical stirrer and condenser. The reaction mixture was slowly heated and refluxed for 3-4 hr. On completion of reaction, monitored by TLC (ethyl acetate:toluene::3:7), the reaction mixture was cooled to room temperature and the solid separated was filtered and washed with mixture of chloroform and methanol to obtain the pure compounds 8a-h and 9a-h. Analytical and spectroscopic characterization data of the synthesized compounds 8a-h and 9a-h are given below:

2-Amino-4-(1,3-diphenyl-1 $\boldsymbol{H}$-pyrazol-4-yl)-4H-benzo[ $\boldsymbol{h}]$ chromene-3-carbonitrile 8a. Yield: 73 \%. m.p. 186-188 ${ }^{\circ}$ C. Anal.Calcd. for $\mathrm{C}_{29} \mathrm{H}_{20} \mathrm{~N}_{4} \mathrm{O}$ (440.51 gm/mole): C 79.07, H 4.58, N 12.72 \%. Found: C 79.26, H 4.44, N $12.53 \%$. IR (KBr, v, $\mathrm{cm}^{-1}$ ): 3400 \& 3295 (asym. \& sym. str. of $\mathrm{NH}_{2}$ ), 2185 (-C $\equiv \mathrm{N}$ str.), 1245 (asym. str. of cyclic ArC-O-C ether). ${ }^{1} \mathrm{H}$ NMR (400 MHz, DMSO$\left.d_{6}\right) \delta_{\mathrm{H}}(\mathrm{ppm}): 4.97(\mathrm{~s}, 1 \mathrm{H}$, pyran $\mathrm{H} 4), 6.91$ (s, 2H, $\mathrm{D}_{2} \mathrm{O}$ exch., $\left.\mathrm{NH}_{2}\right), 7.04-8.29(\mathrm{~m}, 16 \mathrm{H}, \mathrm{Ar}-\mathrm{H})$, 8.31 (pyrazole H5). ${ }^{13} \mathrm{C}$ NMR (100 MHz, DMSO- $d_{6}$ ) $\delta_{\mathrm{C}}(\mathrm{ppm}): 30.4$ (pyran C4), 56.7 (pyran C3), 115.9 (pyran C5), 120.9 (CN), 117.8, 118.8, 122.1, 123.3, 124.4, 125.5, 126.2, 126.3, 127.0, $127.3,128.5,128.6,129.3,129.4,132.8,134.1,136.1,139.1,144.2$ (Ar-ㄷ), 146.1 (pyran C6), 160.7 (pyran C2).

\section{2-Amino-4-(3-(4-bromophenyl)-1-phenyl-1H-pyrazol-4-yl)-4H-benzo[ $h]$ chromene-3-} carbonitrile 8b. Yield: 69 \%. m.p. 231-233 ${ }^{\circ} \mathrm{C}$. Anal.Calcd. for $\mathrm{C}_{29} \mathrm{H}_{19} \mathrm{BrN}_{4} \mathrm{O}(519.40$ gm/mole): C 67.06, H 3.69, N 10.79 \%. Found: C 66.83, H 3.50, N $10.93 \%$. IR (KBr, v, cm $\left.{ }^{-1}\right)$ : 3415 \& 3305 (asym. \& sym. str. of $-\mathrm{NH}_{2}$ ), 2190 (-C $\equiv \mathrm{N}$ str.), 1265 (asym. str. of cyclic ArC-O-C ether). ${ }^{1} \mathrm{H}$ NMR (400 MHz, DMSO- $\left.d_{6}\right) \delta_{\mathrm{H}}(\mathrm{ppm}): 5.15$ (s, $1 \mathrm{H}$, pyran H4), 6.89 (s, 2H, $\mathrm{D}_{2} \mathrm{O}$ exch, $\mathrm{NH}_{2}$ ), 7.12-8.14 (m, 15H, Ar-H), 8.27 (pyrazole H5). ${ }^{13} \mathrm{C}$ NMR (100 MHz, DMSO- $\left.d_{6}\right) \delta_{\mathrm{C}}(\mathrm{ppm})$ : 31.1 (pyran C4), 56.8 (pyran C3), 116.3 (pyran C5), 120.8 (CN), 118.0, 119.0, 123.0, 124.0, $125.7,126.1$, 126.4, 126.5, 127.5, 127.6, 128.2, 128.3, 129.2, 129.7, 131.1, 134.8, 137.8, 139.6, 144.3 (Ar-C), 147.0 (pyran C6), 160.8 (pyran C2).

2-Amino-4-(3-(4-chlorophenyl)-1-phenyl-1H-pyrazol-4-yl)-4H-benzo[ $h]$ chromene-3-carbonitrile 8c. Yield: 67 \%. m.p. 200-202 ${ }^{\circ} \mathrm{C}$. Anal.Calcd. for $\mathrm{C}_{29} \mathrm{H}_{19} \mathrm{ClN}_{4} \mathrm{O}$ (474.95 gm/mole): C 73.34, H 4.03, N 11.80 \%. Found: C 73.22, H 3.86, N $11.99 \%$. IR (KBr, v, cm $\left.{ }^{-1}\right): 3405 \& 3335$ (asym. \& sym. str. of $-\mathrm{NH}_{2}$ ), 2180 (-C $\equiv \mathrm{N}$ str.), 1230 (asym. str. of cyclic ArC-O-C ether). ${ }^{1} \mathrm{H}$ NMR (400 MHz, DMSO-d $\left.d_{6}\right) \delta_{\mathrm{H}}(\mathrm{ppm}): 5.23$ (s, 1H, pyran H4), 6.86 (s, 2H, D $\mathrm{O}_{2}$ exch., $\mathrm{NH}_{2}$ ), 7.07-8.16 (m, 15H, Ar-H), 8.39 (pyrazole H5). ${ }^{13} \mathrm{C}$ NMR (100 MHz, DMSO- $\left.d_{6}\right) \delta_{\mathrm{C}}(\mathrm{ppm}): 30.5$ (pyran C4), 57.5 (pyran C3), 117.0 (pyran C5), 120.9 (CN), 118.7, 119.3, 122.9, 124.9, 125.4, $126.4,126.7,126.8,127.8,128.0,128.1,129.5,129.6,129.9,131.7,134.1,137.6,139.6,143.0$ (Ar-C), 148.9 (pyran C6), 161.4 (pyran C2).

2-Amino-4-(3-(4-fluorophenyl)-1-phenyl-1H-pyrazol-4-yl)-4H-benzo[ $\boldsymbol{h}]$ chromene-3-carbonitrile 8d. Yield: 68 \%. m.p. $204-205^{\circ} \mathrm{C}$. Anal.Calcd. for $\mathrm{C}_{29} \mathrm{H}_{19} \mathrm{FN}_{4} \mathrm{O}(458.50 \mathrm{gm} / \mathrm{mole})$ : C 
75.97, H 4.18, N $12.22 \%$. Found: C 76.20, H 4.37, N $12.15 \%$. IR (KBr, $\left.v, \mathrm{~cm}^{-1}\right): 3455 \& 3310$ (asym. \& sym. str. of $-\mathrm{NH}_{2}$ ), 2195 (-C $\equiv \mathrm{N}$ str.), 1225 (asym. str. of cyclic ArC-O-C ether). ${ }^{1} \mathrm{H}$ NMR (400 MHz, DMSO- $\left.d_{6}\right) \delta_{\mathrm{H}}(\mathrm{ppm}): 5.10$ (s, 1H, pyran H4), 6.94 (s, 2H, $\mathrm{D}_{2} \mathrm{O}$ exch., $\mathrm{NH}_{2}$ ), 7.18-8.25 (m, 15H, Ar-H), 8.47 (pyrazole H5). ${ }^{13} \mathrm{C}$ NMR (100 MHz, DMSO- $\left.d_{6}\right) \delta_{\mathrm{C}}$ (ppm): 29.9 (pyran C4), 58.0 (pyran C3), 115.1 (pyran C5), $121.0(\mathrm{CN}), 117.4,118.7,123.2,124.5,125.3$, 126.0, 126.3, 126.7, 127.1, 127.6, 128.6, 129.2, 129.4, 129.6, 130.0, 134.8, 136.6, 138.4, 144.7 (Ar-C), 150.2 (pyran C6), 160.8 (pyran C2).

2-Amino-4-(3-(4-methoxyphenyl)-1-phenyl-1 $H$-pyrazol-4-yl)-4H-benzo[ $h]$ chromene-3carbonitrile 8e. Yield: $65 \%$. m.p. $239-241{ }^{\circ} \mathrm{C}$. Anal.Calcd. for $\mathrm{C}_{30} \mathrm{H}_{22} \mathrm{~N}_{4} \mathrm{O}_{2}(470.53 \mathrm{gm} / \mathrm{mole})$ : C 76.58, H 4.71, N $11.91 \%$. Found: C 76.43, H 4.40, N $12.16 \%$. IR (KBr, v, $\left.\mathrm{cm}^{-1}\right): 3400$ \& 3290 (asym. \& sym. str. of $-\mathrm{NH}_{2}$ ), 2205 ( $-\mathrm{C} \equiv \mathrm{N}$ str.), 1250 (asym. str. of cyclic ArC-O-C ether). ${ }^{1} \mathrm{H}$ NMR (400 MHz, DMSO- $d_{6}$ ) $\delta_{\mathrm{H}}(\mathrm{ppm}): 3.87\left(\mathrm{~s}, 3 \mathrm{H}, \mathrm{OCH}_{3}\right), 5.22(\mathrm{~s}, 1 \mathrm{H}$, pyran H4), 6.90 (s, $2 \mathrm{H}, \mathrm{D}_{2} \mathrm{O}$ exch., $\mathrm{NH}_{2}$ ), 7.14-8.13 (m, 15H, Ar-H), 8.32 (pyrazole H5). ${ }^{13} \mathrm{C}$ NMR (100 MHz, DMSO- $\left.d_{6}\right) \delta_{\mathrm{C}}(\mathrm{ppm}): 31.0$ (pyran C4), $54.7\left(\mathrm{OCH}_{3}\right), 56.8$ (pyran C3), 115.9 (pyran C5), 120.9 (CN), 117.2, 118.9, 123.9, 124.7, 125.1, 126.1, 126.5, 127.2, 127.8, 128.6, 128.8, 129.0, 129.8, 131.3, 134.7, 137.9, 139.7, 143.9 (Ar-C), 147.6 (pyran C6), $158.0\left(\right.$ Ar $\left.\underline{\mathrm{C}}-\mathrm{OCH}_{3}\right), 162.0$ (pyran $\mathrm{C} 2)$.

2-Amino-4-(1-phenyl-3-(4-methylphenyl)-1 $H$-pyrazol-4-yl)-4H-benzo[ $h]$ chromene-3-carbonitrile 8f. Yield: $63 \%$ m.p. $199-201{ }^{\circ} \mathrm{C}$. Anal.Calcd. for $\mathrm{C}_{30} \mathrm{H}_{22} \mathrm{~N}_{4} \mathrm{O}(454.53 \mathrm{gm} / \mathrm{mole})$ : C 79.28, H 4.88, N $12.33 \%$. Found: C 79.11, H 5.04, N $12.12 \%$. IR (KBr, $\left.v, \mathrm{~cm}^{-1}\right): 3395 \& 3320$ (asym. \& sym. str. of $-\mathrm{NH}_{2}$ ), 2215 (-C $\equiv \mathrm{N}$ str.), 1215 (asym. str. of cyclic ArC-O-C ether). ${ }^{1} \mathrm{H}$ NMR (400 MHz, DMSO- $\left.d_{6}\right) \delta_{\mathrm{H}}(\mathrm{ppm}): 2.31\left(\mathrm{~s}, 3 \mathrm{H}, \mathrm{CH}_{3}\right), 5.19(\mathrm{~s}, 1 \mathrm{H}$, pyran H4), $6.96(\mathrm{~s}, 2 \mathrm{H}$, $\mathrm{D}_{2} \mathrm{O}$ exch., $\mathrm{NH}_{2}$ ), 6.98-8.24 (m, 15H, Ar-H), 8.47 (pyrazole H5). ${ }^{13} \mathrm{C}$ NMR (100 MHz, DMSO$\left.d_{6}\right) \delta_{\mathrm{C}}(\mathrm{ppm}): 21.2\left(\mathrm{CH}_{3}\right), 31.2$ (pyran $\left.\mathrm{C} 4\right), 56.5$ (pyran $\mathrm{C} 3$ ), 117.9 (pyran $\left.\mathrm{C} 5\right), 121.0(\mathrm{CN})$, 118.4, 121.2, 123.2, 124.2, 126.2, 126.3, 126.5, 127.0, 127.1, 128.0, 128.6, 128.9, 129.3, 129.9, 130.6, 133.2, 137.7, 139.8, 142.9 (Ar-C), 151.6 (pyran C6), 160.2 (pyran C2). MS: 455.1 $\left(\mathrm{M}^{+}+1\right)$.

2-Amino-4-(3-(4-nitrophenyl)-1-phenyl-1 $H$-pyrazol-4-yl)-4 $H$-benzo[ $h]$ chromene-3-carbonitrile 8g. Yield: $65 \%$. m.p. $224-226{ }^{\circ} \mathrm{C}$. Anal.Calcd. for $\mathrm{C}_{29} \mathrm{H}_{19} \mathrm{~N}_{5} \mathrm{O}_{3}(485.51 \mathrm{gm} / \mathrm{mole})$ : C 71.74, H 3.95, N $14.43 \%$. Found: C 71.89, H 4.14, N $14.30 \%$. IR $\left(\mathrm{KBr}, v, \mathrm{~cm}^{-1}\right): 3430 \& 3315$ (asym. \& sym. str. of $-\mathrm{NH}_{2}$ ), 2220 (-C $\equiv \mathrm{N}$ str.), 1260 (asym. str. of cyclic ArC-O-C ether). ${ }^{1} \mathrm{H}$ NMR (400 MHz, DMSO- $\left.d_{6}\right) \delta_{\mathrm{H}}(\mathrm{ppm}): 5.36(\mathrm{~s}, 1 \mathrm{H}$, pyran $\mathrm{H} 4), 6.87$ (s, $2 \mathrm{H}, \mathrm{D}_{2} \mathrm{O}$ exch., $\mathrm{NH}_{2}$ ), 7.02-8.17 (m, 10H, Ar-H), 8.21 (pyrazole H5). ${ }^{13} \mathrm{C}$ NMR (100 MHz, DMSO- $\left.d_{6}\right) \delta_{\mathrm{C}}(\mathrm{ppm}): 31.2$ (pyran C4), 58.1 (pyran C3), 115.9 (pyran C5), 121.1 (CN), 117.2, 118.6, 123.9, 124.1, 125.4, 126.4, 126.5, 126.9, 127.3, 127.5, 128.0, 128.2, 129.4, 129.6, 130.5, 133.3, 136.4, 139.3, 142.7 (Ar-C), 147.8 (pyran C6), 161.5 (pyran C2).

2-Amino-4-(1-phenyl-3-(thien-2-yl)-1 $\boldsymbol{H}$-pyrazol-4-yl)-4 $\boldsymbol{H}$-benzo[ $\boldsymbol{h}$ ]chromene-3-carbonitrile 8h. Yield: $60 \%$. m.p. $237-238{ }^{\circ} \mathrm{C}$. Anal.Calcd. for $\mathrm{C}_{27} \mathrm{H}_{18} \mathrm{~N}_{4} \mathrm{OS}$ (446.54 gm/mole): $\mathrm{C} 72.63, \mathrm{H}$ 4.06, N $12.55 \%$. Found: C 72.35, H 3.90, N $12.70 \%$. IR (KBr, $\left.v, \mathrm{~cm}^{-1}\right): 3435 \& 3345$ (asym. \& sym. str. of $-\mathrm{NH}_{2}$ ), 2185 (-C $\equiv \mathrm{N}$ str.), 1235 (asym. str. of cyclic ArC-O-C ether). ${ }^{1} \mathrm{H}$ NMR (400 
MHz, DMSO- $\left.d_{6}\right) \delta_{\mathrm{H}}(\mathrm{ppm}): 5.29$ (s, $1 \mathrm{H}$, pyran H4), $6.92\left(\mathrm{~s}, 2 \mathrm{H}, \mathrm{D}_{2} \mathrm{O}\right.$ exch., $\left.\mathrm{NH}_{2}\right)$, 7.20-7.98 (m, $14 \mathrm{H}, \mathrm{Ar}-\mathrm{H}$ ), 8.33 (pyrazole H5). ${ }^{13} \mathrm{C}$ NMR (100 MHz, DMSO- $\left.d_{6}\right) \delta_{\mathrm{C}}(\mathrm{ppm}): 29.8$ (pyran C4), 57.4 (pyran C3), 116.5 (pyran C5), $121.0(\mathrm{CN}), 117.3,118.3,122.4,124.4,125.0,126.4,126.8$, $127.0,127.2,128.1,128.5,128.5,129.3,129.9,130.2,134.1,137.5,139.2,142.5$ (Ar-C), 150.3 (pyran C6), 161.4 (pyran C2).

3-Amino-1-(1,3-diphenyl-1 $H$-pyrazol-4-yl)-1 $H$-benzo[f]chromene-2-carbonitrile (9a). Yield: 76 \%. m.p. $255-257{ }^{\circ} \mathrm{C}$. Anal.Calcd. for $\mathrm{C}_{29} \mathrm{H}_{20} \mathrm{~N}_{4} \mathrm{O}$ (440.51 gm/mole): C 79.07, H 4.58, N 12.72 \%. Found: C 79.18, H 4.41, N $12.81 \%$. IR (KBr, v, $\mathrm{cm}^{-1}$ ): 3450 \& 3290 (asym. \& sym. str. of $\mathrm{NH}_{2}$ ), 2180 (-C $\equiv \mathrm{N}$ str.), 1270 (asym. str. of cyclic ArC-O-C ether). ${ }^{1} \mathrm{H}$ NMR (400 MHz, DMSO$\left.d_{6}\right) \delta_{\mathrm{H}}(\mathrm{ppm}):{ }^{1} \mathrm{H}$ NMR (400 MHz, DMSO-d $) \delta_{\mathrm{H}}(\mathrm{ppm}): 5.19$ (s, 1H, pyran H4), 6.98 (s, 2H, $\mathrm{D}_{2} \mathrm{O}$ exch., $\mathrm{NH}_{2}$ ), 7.00-8.22 (m, 16H, Ar-H), 8.51 (pyrazole H5). ${ }^{13} \mathrm{C}$ NMR (100 MHz, DMSO$\left.d_{6}\right) \delta_{\mathrm{C}}(\mathrm{ppm}): 29.8$ (pyran C4), 57.2 (pyran C3), 116.8 (pyran C5), $121.2(\mathrm{CN}), 117.1,118.9$, $122.7,124.6,125.6,126.7,126.9$, 127.2, 127.2, 128.0, 128.5, 129.4, 129.5, 129.7, 130.9, 133.3, 137.4, 138.7, 144.5 (Ar-C), 147.7 (pyran C6), 160.7 (pyran C2).

3-Amino-1-(3-(4-bromophenyl)-1-phenyl-1 $H$-pyrazol-4-yl)-1 $\boldsymbol{H}$-benzo[ $\boldsymbol{f}]$ chromene-2-carbonitrile 9b. Yield: $58 \%$. m.p. $221-223{ }^{\circ} \mathrm{C}$. Anal Anal.Calcd. for $\mathrm{C}_{29} \mathrm{H}_{19} \mathrm{BrN}_{4} \mathrm{O}(519.40 \mathrm{gm} / \mathrm{mole})$ : C 67.06, H 3.69, N 10.79 \%. Found: C 66.93, H 3.80, N $10.97 \%$. IR (KBr, v, cm $\left.{ }^{-1}\right): 3425$ \& 3290 (asym. \& sym. str. of $\left.-\mathrm{NH}_{2}\right), 2200$ ( $\mathrm{C} \equiv \mathrm{N}$ str.), 1240 (asym. str. of cyclic ArC-O-C ether). ${ }^{1} \mathrm{H}$ NMR (400 MHz, DMSO- $\left.d_{6}\right) \delta_{\mathrm{H}}(\mathrm{ppm}):{ }^{1} \mathrm{H}$ NMR $\left(400 \mathrm{MHz}, \mathrm{DMSO}-d_{6}\right) \delta_{\mathrm{H}}(\mathrm{ppm}): 5.37$ (s, $1 \mathrm{H}$, pyran H4), 6.90 (s, 2H, $\mathrm{D}_{2} \mathrm{O}$ exch., $\mathrm{NH}_{2}$ ), 6.96-8.18 (m, 15H, Ar-H), 8.41 (pyrazole H5). ${ }^{13} \mathrm{C}$ NMR (100 MHz, DMSO- $\left.d_{6}\right) \delta_{\mathrm{C}}(\mathrm{ppm}): 30.5$ (pyran C4), 57.3 (pyran C3), 116.7 (pyran C5), 120.8 (CN), 117.2, 118.8, 123.0, 124.1, 125.0, 126.3, 126.6, 127.3, 127.7, 128.1, 128.6, 128.9, 129.4, 129.7, 131.6, 133.8, 136.2, 138.0, 143.8 (Ar-C), 148.5 (pyran C6), 162.0 (pyran C2).

3-Amino-1-(3-(4-chlorophenyl)-1-phenyl-1H-pyrazol-4-yl)-1H-benzo[f $\mathbf{H}$ chromene-2-carbonitrile 9c. Yield: $62 \%$. m.p. $210-212{ }^{\circ} \mathrm{C}$. Anal.Calcd. for $\mathrm{C}_{29} \mathrm{H}_{19} \mathrm{ClN}_{4} \mathrm{O}(474.95 \mathrm{gm} / \mathrm{mole})$ : C 73.34, H 4.03, N 11.80 \%. Found: C 73.58, H 3.92, N $11.93 \%$. IR (KBr, v, cm $\left.{ }^{-1}\right): 3455$ \& 3295 (asym. \& sym. str. of $-\mathrm{NH}_{2}$ ), 2190 (-C $\equiv \mathrm{N}$ str.), 1275 (asym. str. of cyclic ArC-O-C ether). ${ }^{1} \mathrm{H}$ NMR (400 MHz, DMSO- $\left.d_{6}\right) \delta_{\mathrm{H}}(\mathrm{ppm}): 5.25$ (s, 1H, pyran H4), $6.88\left(\mathrm{~s}, 2 \mathrm{H}, \mathrm{D}_{2} \mathrm{O}\right.$ exch., $\mathrm{NH}_{2}$ ), 7.05-8.20 (m, 15H, Ar-H), 8.35 (pyrazole H5). ${ }^{13} \mathrm{C}$ NMR (100 MHz, DMSO- $\left.d_{6}\right) \delta_{\mathrm{C}}(\mathrm{ppm}): 30.6$ (pyran C4), 58.1 (pyran C3), 115.8 (pyran C5), $120.8(\mathrm{CN}), 117.8,118.1,122.3,124.4,125.9$, 126.2 , 126.4, 126.5, 127.6, 127.8, 128.7, 128.8, 129.2, 129.3, 131.7, 134.1, 137.2, 139.4, 144.6 (Ar-C), 148.4 (pyran C6), 160.8 (pyran C2).

3-Amino-1-(3-(4-fluorophenyl)-1-phenyl-1H-pyrazol-4-yl)-1H-benzo[f $]$ chromene-2-carbonitrile 9d. Yield: 65 \%. m.p. 252-254 ${ }^{\circ} \mathrm{C}$. Anal.Calcd. for $\mathrm{C}_{29} \mathrm{H}_{19} \mathrm{FN}_{4} \mathrm{O}$ (458.50 gm/mole): C 75.97, H 4.18, N $12.22 \%$. Found: C 76.09, H 4.06, N $12.10 \%$. IR (KBr, v, cm $\left.{ }^{-1}\right): 3400 \& 3300$ (asym. \& sym. str. of $-\mathrm{NH}_{2}$ ), 2210 (-C $\equiv \mathrm{N}$ str.), 1255 (asym. str. of cyclic ArC-O-C ether). ${ }^{1} \mathrm{H}$

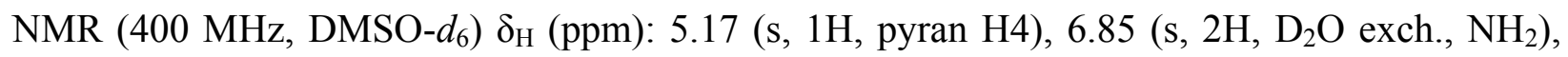
7.10-7.99 (m, 15H, Ar-H), 8.45 (pyrazole H5). ${ }^{13} \mathrm{C}$ NMR (100 MHz, DMSO- $\left.d_{6}\right) \delta_{\mathrm{C}}(\mathrm{ppm}): 31.1$ (pyran C4), 56.9 (pyran C3), 117.4 (pyran C5), $121.1(\mathrm{CN}), 118.0,119.9,123.4,124.3,125.4$, 
$126.3,126.7,127.4,127.5,127.9,128.1,128.4,129.3,129.4,130.8,133.3,136.4,139.3,143.1$ (Ar-C), 149.6 (pyran C6), 161.3 (pyran C2).

3-Amino-1-(3-(4-methoxyphenyl)-1-phenyl-1H-pyrazol-4-yl)-1H-benzo[f]chromene-2carbonitrile 9e. Yield: 69 \%. m.p. $194-195{ }^{\circ} \mathrm{C}$. Anal.Calcd. for $\mathrm{C}_{30} \mathrm{H}_{22} \mathrm{~N}_{4} \mathrm{O}_{2}(470.53 \mathrm{gm} / \mathrm{mole})$ : C 76.58, H 4.71, N $11.91 \%$. Found: C 76.67, H 4.56, N $12.03 \%$. IR (KBr, v, cm $\left.{ }^{-1}\right): 3415$ \& 3280 (asym. \& sym. str. of $-\mathrm{NH}_{2}$ ), 2195 (-C $\equiv \mathrm{N}$ str.), 1220 (asym. str. of cyclic ArC-O-C ether). ${ }^{1} \mathrm{H}$ NMR (400 MHz, DMSO- $\left.d_{6}\right) \delta_{\mathrm{H}}(\mathrm{ppm}): 3.94\left(\mathrm{~s}, 3 \mathrm{H}, \mathrm{OCH}_{3}\right), 5.32$ (s, 1H, pyran H4), 6.93 (s, $2 \mathrm{H}, \mathrm{D}_{2} \mathrm{O}$ exch., $\mathrm{NH}_{2}$ ), 7.01-8.11 (m, 15H, Ar-H), 8.27 (pyrazole H5). ${ }^{13} \mathrm{C}$ NMR (100 MHz, DMSO- $\left.d_{6}\right) \delta_{\mathrm{C}}(\mathrm{ppm}): 29.9$ (pyran $\left.\mathrm{C} 4\right), 55.8\left(\mathrm{OCH}_{3}\right), 58.7$ (pyran $\mathrm{C} 3$ ), 117.4 (pyran $\left.\mathrm{C} 5\right), 121.1$ (CN), 118.3, 119.5, 123.2, 124.2, 125.5, 126.1, 126.3, 127.1, 127.6, 128.5, 128.7, 129.0, 129.6, 131.3, 133.3, 136.2, 138.0, 144.4 (Ar-C), 149.6 (pyran C6), $157.4\left(\right.$ ArC $\left.-\mathrm{OCH}_{3}\right), 161.3$ (pyran C2).

3-Amino-1-(1-phenyl-3-(4-methylphenyl)-1H-pyrazol-4-yl)-1H-benzo[f]chromene-2-

carbonitrile 9f. Yield: $62 \%$. m.p. $279-281{ }^{\circ} \mathrm{C}$. Anal.Calcd. for $\mathrm{C}_{30} \mathrm{H}_{22} \mathrm{~N}_{4} \mathrm{O}(454.53 \mathrm{gm} / \mathrm{mole})$ : C 79.28, H 4.88, N 12.33 \%. Found: C 79.08, H 4.98, N $12.51 \%$. IR (KBr, v, cm $\left.{ }^{-1}\right): 3460$ \& 3310 (asym. \& sym. str. of $\left.-\mathrm{NH}_{2}\right), 2205$ ( $\mathrm{C} \equiv \mathrm{N}$ str.), 1235 (asym. str. of cyclic ArC-O-C ether). ${ }^{1} \mathrm{H}$ NMR (400 MHz, DMSO- $\left.d_{6}\right) \delta_{\mathrm{H}}(\mathrm{ppm}): 2.37$ (s, 3H, $\left.\mathrm{CH}_{3}\right), 5.24$ (s, 1H, pyran H4), 6.84 (s, $2 \mathrm{H}, \mathrm{D}_{2} \mathrm{O}$ exch., $\mathrm{NH}_{2}$ ), 6.96-8.19 (m, 15H, Ar-H), 8.32 (pyrazole H5). ${ }^{13} \mathrm{C}$ NMR (100 MHz, DMSO- $\left.d_{6}\right) \delta_{\mathrm{C}}(\mathrm{ppm}): 21.4\left(\mathrm{CH}_{3}\right), 29.4$ (pyran C4), 57.2 (pyran C3), 116.7 (pyran C5), 120.9 (CN), 118.2, 119.0, 122.6, 123.1, 123.4, 124.2, 125.5, 126.1, 127.5, 128.1, 128.3, 129.1, 129.4, 129.8, 131.2, 134.1, 138.1, 139.7, 142.1 (Ar-C), 148.9 (pyran C6), 160.0 (pyran C2).

3-Amino-1-(3-(4-nitrophenyl)-1-phenyl-1H-pyrazol-4-yl)-1 $\boldsymbol{H}$-benzo[f]chromene-2-

carbonitrile 9g. Yield: $60 \%$. m.p. $238-240{ }^{\circ} \mathrm{C}$. Anal.Calcd. for $\mathrm{C}_{29} \mathrm{H}_{19} \mathrm{~N}_{5} \mathrm{O}_{3}(485.51 \mathrm{gm} / \mathrm{mole})$ : C 71.74, H 3.95, N 14.43 \%. Found: C 71.96, H 3.75, N $14.28 \%$. IR (KBr, v, cm $\left.{ }^{-1}\right): 3420$ \& 3295 (asym. \& sym. str. of $\left.-\mathrm{NH}_{2}\right), 2210$ ( $\mathrm{C} \equiv \mathrm{N}$ str.), 1230 (asym. str. of cyclic ArC-O-C ether). ${ }^{1} \mathrm{H}$ NMR (400 MHz, DMSO- $\left.d_{6}\right) \delta_{\mathrm{H}}(\mathrm{ppm}): 5.41$ (s, 1H, pyran H4), 6.97 (s, 2H, D $2 \mathrm{O}$ exch., $\left.\mathrm{NH}_{2}\right)$, 7.05-8.21 (m, 15H, Ar-H), 8.42 (pyrazole H5). ${ }^{13} \mathrm{C}$ NMR (100 MHz, DMSO- $\left.d_{6}\right) \delta_{\mathrm{C}}(\mathrm{ppm}): 30.7$ (pyran C4), 56.8 (pyran C3), 116.3 (pyran C5), 121.2 (CN), 117.7, 118.9, 122.8, 123.1, 125.7, 126.2, 126.7, 127.6, 128.4, 128.6, 128.8, 129.1, 129.3, 129.9, 130.1, 134.9, 137.9, 139.1, 143.2 (Ar-C), 150.1 (pyran C6), 161.4 (pyran C2).

3-Amino-1-(1-phenyl-3-(thien-2-yl)-1H-pyrazol-4-yl)-1H-benzo[f]chromene-2-carbonitrile

9h. Yield: $63 \%$. m.p. $241-243{ }^{\circ} \mathrm{C}$. Anal.Calcd. for $\mathrm{C}_{27} \mathrm{H}_{18} \mathrm{~N}_{4} \mathrm{OS}(446.54 \mathrm{gm} / \mathrm{mole})$ : $\mathrm{C} 72.63, \mathrm{H}$ 4.06, N 12.55 \%. Found: C 72.97, H 3.95, N $12.67 \%$. IR (KBr, v, cm ${ }^{-1}$ ): 3405 \& 3325 (asym. \& sym. str. of $-\mathrm{NH}_{2}$ ), 2200 (-C $\equiv \mathrm{N}$ str.), 1240 (asym. str. of cyclic ArC-O-C ether). ${ }^{1} \mathrm{H}$ NMR (400 $\left.\mathrm{MHz}, \mathrm{DMSO}-d_{6}\right) \delta_{\mathrm{H}}(\mathrm{ppm}): 5.57(\mathrm{~s}, 1 \mathrm{H}$, pyran $\mathrm{H} 4), 6.99\left(\mathrm{~s}, 2 \mathrm{H}, \mathrm{D}_{2} \mathrm{O}\right.$ exch., $\left.\mathrm{NH}_{2}\right), 7.23-7.91(\mathrm{~m}$, 14H, Ar-H), 8.25 (pyrazole H5). ${ }^{13} \mathrm{C}$ NMR (100 MHz, DMSO- $d_{6}$ ) $\delta_{\mathrm{C}}(\mathrm{ppm}): 29.8$ (pyran C4), 57.5 (pyran C3), 115.7 (pyran C5), $121.1(\mathrm{CN}), 117.5,118.4,123.3,125.2,126.6,126.8,127.1$, 127.3, 127.4, 128.3, 128.8, 129.0, 129.8, 130.5, 131.2, 134.8, 137.4, 139.3, 144.7 (Ar-C), 146.6 (pyran C6), 160.2 (pyran C2). MS: $447.0\left(\mathrm{M}^{+}+1\right)$. 


\section{Acknowledgements}

The authors are thankful to Professor \& Head, Department of Chemistry, Sardar Patel University for providing ${ }^{1} \mathrm{H}$ NMR, ${ }^{13} \mathrm{C}$ NMR spectroscopy and research facilities. We are also thankful to Oxygen Healthcare Research Pvt. Ltd., Ahmedabad, for providing mass spectrometry facilities, SICART, Vallabh Vidyanagar, for the FT-IR and elemental analysis and Dhanji P. Rajani, Microcare Laboratory, Surat, for antimicrobial screening of the compounds reported herein. One of the authors is grateful to UGC, New Delhi for a Research Fellowship in Sciences for Meritorious Students.

\section{References}

1. Khafagy, M. M.; El-Wahas, A. H. F. A.; Eid, F. A.; El-Agrody, A. M. Farmaco 2002, 57, 715.

2. (a) Smith, W. P.; Sollis, L. S.; Howes, D. P.; Cherry, C. P.; Starkey, D. I.; Cobley, N. K.; Weston, H.; Scicinski, J.; Merritt, A.; Whittington, A.; Wyatt, P.; Taylor, N.; Green, D.; Bethell, R.; Madar, S.; Fenton, R. J.; Morley, P. J.; Pateman, T.; Beresford, A. J. Med. Chem. 1998, 41, 787. (b) Martinez, A. G.; Marco, L. J. Bioorg. Med. Chem. Lett. 1997, 7, 3165 .

3. Hiramoto, K.; Nasuhara, A.; Michiloshi, K.; Kato, T.; Kikugawa, K. Mutat. Res. 1997, 395, 47.

4. Dell, C. P.; Smith, C. W. Eur. Pat. Appl. 537,949, 1993; Chem. Abstr. 1993, 119, 139102d.

5. Bianchi, G.; Tava, A. Agric. Biol. Chem. 1987, 51, 2001.

6. Mohr, S. J.; Chirigos, M. A.; Fuhrman, F. S.; Pryor, J. W. Cancer Res. 1975, 35, 3750.

7. (a) Skommer, J.; Wlodkowic, D.; Matto, M.; Eray, M.; Pelkonen, J. Leukemia Res. 2006, 30, 322 and references cited therein. (b) Anderson, D. R.; Hegde, S.; Reinhard, E.; Gomez, L.; Vernier, W. F.; Lee, L.; Liu, S.; Sambandam, A.; Snider, P. A.; Masih, L. Bioorg. Med. Chem. Lett. 2005, 15, 1587. (c) Wang, J. L.; Liu, D.; Zhang, Z.; Shan, S.; Han, X.; Srinvasula, S. M.; Croce, C. M.; Alnemeri, E. S.; Huang, Z. Proc. Natl. Acad. Sci. U.S.A. 2000, 97, 7124.

8. Eiden, F.; Denk, F. Arch. Pharm. Weinhein Ger (Arch. Pharm.) 1991, 324, 353.

9. Hafez, E. A. A.; Elnagdi, M. H.; Elagamey, A. G. A.; Ei-Taweel, F. M. A. A. Heterocycles 1987, 26, 903.

10. (a) Damljanovic, I.; Colovic, M.; Vukicevic, M.; Manojlovic, D.; Radulovic, N.; Wurst, K.; Laus, G.; Ratkovic, Z.; Joksovic, M. D.; Vukicevic, R. D. J. Organomet. Chem. 2009, 694, 1575. (b) Damljanovic, I.; Vukicevic, M.; Radulovic, N.; Palic, R.; Ellmerer, E.; Ratkovic, Z.; Joksovic, M. D.; Vukicevic, R. D. Bioorg. Med. Chem. Lett. 2009, 19, 1093. (c) Prakash, O.; Kumar, R.; Parkash, V. Eur. J. Med. Chem. 2008, 43, 435. (d) Prakash, O.; Kumar, R.; Sehrawat, R. Eur. J. Med. Chem. 2009, 44, 1763. 
11. (a) Bekhit, A. A.; Ashour, H. M. A.; Ghany, Y. S. A.; Bekhit, A. E. A.; Baraka, A. M. Eur. J. Med. Chem. 2008, 43, 456. (b) Bekhit, A. A.; Abdel-Aziem, T. Bioorg. Med. Chem. 2004, 12, 1935. (c) Bekhit, A. A.; Fahmy, H. T. Y.; Rostom, S. A. F.; Baraka, A. M. Eur. J. Med. Chem. 2003, 38, 27. (d) Bekhit, A. A.; Fahmy, H. T. Y. Arch. Pharm. Pharm. Med. Chem. 2000, 333, 53.

12. Chovatia, P. T.; Akabari, J. D.; Kachhadia, P. K.; Zalawadia, P. D.; Joshi, H. S. J. Serb. Chem. Soc. 2007, 71, 713.

13. Fahmy, H. T. Y.; Rostom, S. A. F.; Bekhit, A. A. Arch. Pharm. Pharm. Med. Chem. 2002, $335,213$.

14. Abadi, A. H.; Eissa, A. A. H.; Hassan, G. S. Chem. Pharm. Bull. 2003, 51, 838.

15. Rathelot, P.; Azas, N.; El-Kashef, H.; Delmas, F.; Giorgio, C. D.; Timon-David , P.; Maldonado, J.; Vanelle, P. Eur. J. Med. Chem. 2002, 37, 671.

16. (a) Hashem, A. I.; Youssef, A. S. A.; Kandeel, K. A.; Abou-Elmagd, W. S. I. Eur. J. Med. Chem. 2007, 42, 934. (b) Farghaly, A.; El-Kashef, H. Arkivoc 2006, (xi), 76. (c) Farghaly, A.; De Clercq, E.; El-Kashef, H. Arkivoc 2006, (x), 137.

17. (a) Mekheimer, R. A.; Sadek, K. U. J. Het. Chem. 2009, 46, 149. (b) Balalaie, S.; Ramezanpour, S.; Bararjanian, M.; Gross, J. H. Synth. Commun. 2008, 38, 1078. (c) Heravi, M. M.; Baghernejad, B.; Oskooie, H. A. J. Chin. Chem. Soc. 2008, 55, 659. (d) Heravi, M. M.; Bakhtiari, K.; Zadsirjan, V.; Bamoharram, F. F. Heravi, O. M. Bioorg. Med. Chem. Lett. 2007, 17, 4262. (e) Balalaie, S.; Bararjanian, M.; Sheikh-Ahmadi, M. Synth. Commun. 2007, 37, 1097. (f) Fotouhi, L.; Heravi, M. M.; Fatehi, A.; Bakhtiari, K. Tetrahedron Lett. 2007, 48, 5379. (g) Balalaie, S.; Sheikh-Ahmadi, M.; Bararjanian, M. Cat. Commun. 2007, 8, 1724. (h) Balalaie, S.; Bararjanian, M.; Mohammad, A.; Movassagh, B. Synlett 2006, 263. (i) Rong, L.; Li, X.; Wang, H.; Shi, D.; Tu, S.; Zhuang, Q. Synth. Commun. 2006, 36, 2363. (j) Maggi, R.; Ballini, R.; Sartori, G.; Sartorio, R. Tetrahedron Lett. 2004, 45, 2297. (k) Wang, X. S.; Shi, D. Q.; Yu, H. Z.; Wang, G. F.; Tu, S. J. Synth. Commun. 2004, 34, 509. (1) Ballini, R.; Bosica, G.; Conforti, M. L.; Maggi, R.; Mazzacani, A.; Righi, P.; Sartori, G. Tetrahedron 2001, 57, 1395. (m) Ballini, R.; Bigi, F.; Conforti, M. L.; Catal. Today 2000, $60,305$.

18. (a) Thumar, N. J.; Patel, M. P. Phosphorus, Sulfur, Silicon. 2009, 184, 2720. (b) Shah, N. K.; Patel, M. P.; Patel, R. G. Phosphorus, Sulfur, Silicon. 2009, 184, 2704. (c) Ladani, N. K.; Patel, M. P.; Patel, R. G. Arkivoc 2009, (vii), 292. (d) Shah, N. K.; Patel, M. P.; Patel, R. G. Indian J. Chem. 2009, 48B, 1170. (e) Nirmal, J. P.; Patel, M. P.; Patel, R. G. Indian J. Chem. 2009, 48B, 712. (f) Ladani, N. K.; Patel, M. P.; Patel, R. G. Indian J. Chem. 2009, 48B, 261. (g) Patel, N. A.; Patel, M. P.; Patel, R. G. J. Environ. Res. Dev. 2009, 3, 851. (g) Thakor, S. F.; Parmar, P. V.; Patel, M. P.; Patel, R. G. Saudi Pharma. J. 2008, 16, 64. (h) Patel, N. A.; Patel, M. P.; Patel, R. G. Phosphorus, Sulfur, Silicon. 2008, 183, 2191. (i) Thakor, S. F.; Patel, D. M..; Patel, M. P.; Patel, R. G. Saudi Pharma. J. 2007, 15, 48. (j) Patel, B. B.; Patel, M. P.; Patel, R. G. J. Serb. Chem. Soc. 2006, 71, 1015. (k) Patel, R. D.; Patel, M. P.; Patel, R. G. Indian J. Chem. 2005, 44B, 1944. 
19. National Committee for Clinical Laboratory Standards (NCCLS), 940, West Valley Road, Suite 1400, Wayne, Pennsylvania 19087-1898, USA. Performance Standards for Antimicrobial Susceptibility Testing; Twelfth Informational Supplement (ISBN 1-56238454 -6), 2002, M100-S12 (M7).

20. Kira, M. A.; Abdel-Rahman, M. O.; Gadalla, K. Z. Tetrahedron Lett. 1969, 10, 109. 